\title{
Light-Activatable Assembled Nanoparticles to Improve Tumor-Penetration and Eradicate Metastasis in Triple Negative Breast Cancer
}

\author{
Ji, Jianfeng
}

2018-08-15

Ji , J , Ma , F , Zhang , H , Liu , F , He , J , Li , W , Xie , T , Zhong , D , Zhang , T , Tian , M , Zhang , H , Almeida Santos, H \& Zhou , M 2018 , ' Light-Activatable Assembled Nanoparticles to Improve Tumor-Penetration and Eradicate Metastasis in Triple Negative Breast Cancer ' , Advanced Functional Materials , vol. 28 , no. 33 , 1801738 . https://doi.org/10.1002/adfm.20180173

http://hdl.handle.net/10138/327320

https://doi.org/10.1002/adfm.201801738

acceptedVersion

Downloaded from Helda, University of Helsinki institutional repository.

This is an electronic reprint of the original article.

This reprint may differ from the original in pagination and typographic detail.

Please cite the original version. 


\section{Advanced Functional Materials \\ Light-Activatable Assembled Nanoparticles to Improve Tumor-Penetration and Eradicate Metastasis in Triple Negative Breast Cancer --Manuscript Draft--}

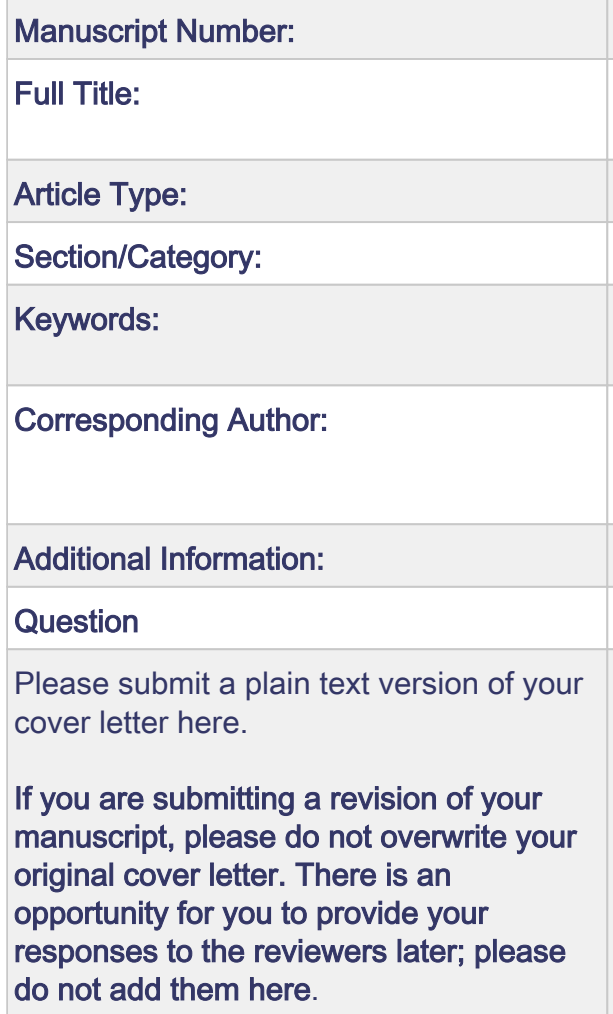

adfm.201801738R2

Light-Activatable Assembled Nanoparticles to Improve Tumor-Penetration and Eradicate Metastasis in Triple Negative Breast Cancer

Full Paper

triple-negative breast cancer; Nanoparticles; self-assembly; Tumor penetration; cancer metastasis

Helder Santos, D.Sc. (Chem. Eng.)

University of Helsinki

Helsinki, Helsinki FINLAND

Additional Information:

Please submit a plain text version of your

Response

March 05, 2018

Editor-in-Chief: Joern Ritterbusch

Dear Dr. Joern Ritterbusch,

On behalf of all co-authors, I would like to submit our manuscript entitled "Non-

Cardiotoxic Tumor-Penetrating Nanosystem for Triple Negative Breast Cancer to

Eradicate Lung and Liver Metastasis" for publication in Advanced Functional Materials.

Background:

Triple-negative breast cancer (TNBC) is highly aggressive combining with fast metastatic behavior, which has the lowest survival rate among all breast cancer types. Doxorubicin (DOX) is one of the most used in breast cancer patients after surgery. However, severe cardiotoxicity and poor tumor penetration depth, for DOX relative treatment to hinder its clinical practice. Furthermore, DOX relative treatment cannot restrain the metastasis of TNBC and inhibit tumor recurrences. Therefore, a rational, complementing chemotherapeutic agent would reduce drug dosage to overcome cardiotoxicity, improve tumor perfusion to maintain intratumoral drug delivery, and prevent the tumor metastasis is necessary.

Our solution:

In this work, our groups have resolved these important problems and report developed a novel nanosystem by a designed assembly method to overcome tumor penetration depth, tumor metastasis, and cardiotoxicity. We predicted that the large $(\sim 36 \mathrm{~nm})$ selfassembled temperature sensitive nanoclusters (TSNCs) could possess good blood persistence and preferential tumor accumulation due to their EPR effect. The TSNCs could be disassembled into ultrasmall nanodots $(\sim 6 \mathrm{~nm})$ under the near-infrared (NIR) laser irradiation, accelerating the deep penetration in the intratumoral environment.

\section{Significance:}

There are multiple major advances in our work and include:

the nanosystem can significantly improve the drug penetration depth under NIR laser exposure due to their changeable particle size;

the nanosystem can eradicate breast Cancer Stem Cells and then could inhibit lung and liver metastasis of TNBC;

Non-cardiotoxicity was evaluated by echocardiography image after treatment;

The treatment can be guided by PET and photoacoustic dual-imaging.

Nanoscale device such as this may overcome one of the major concerns associated with the clinical translation of functional nanoparticles, which is cardiotoxic effect of DOX. The nanoparticle platform has broad preclinical and clinical implications. 


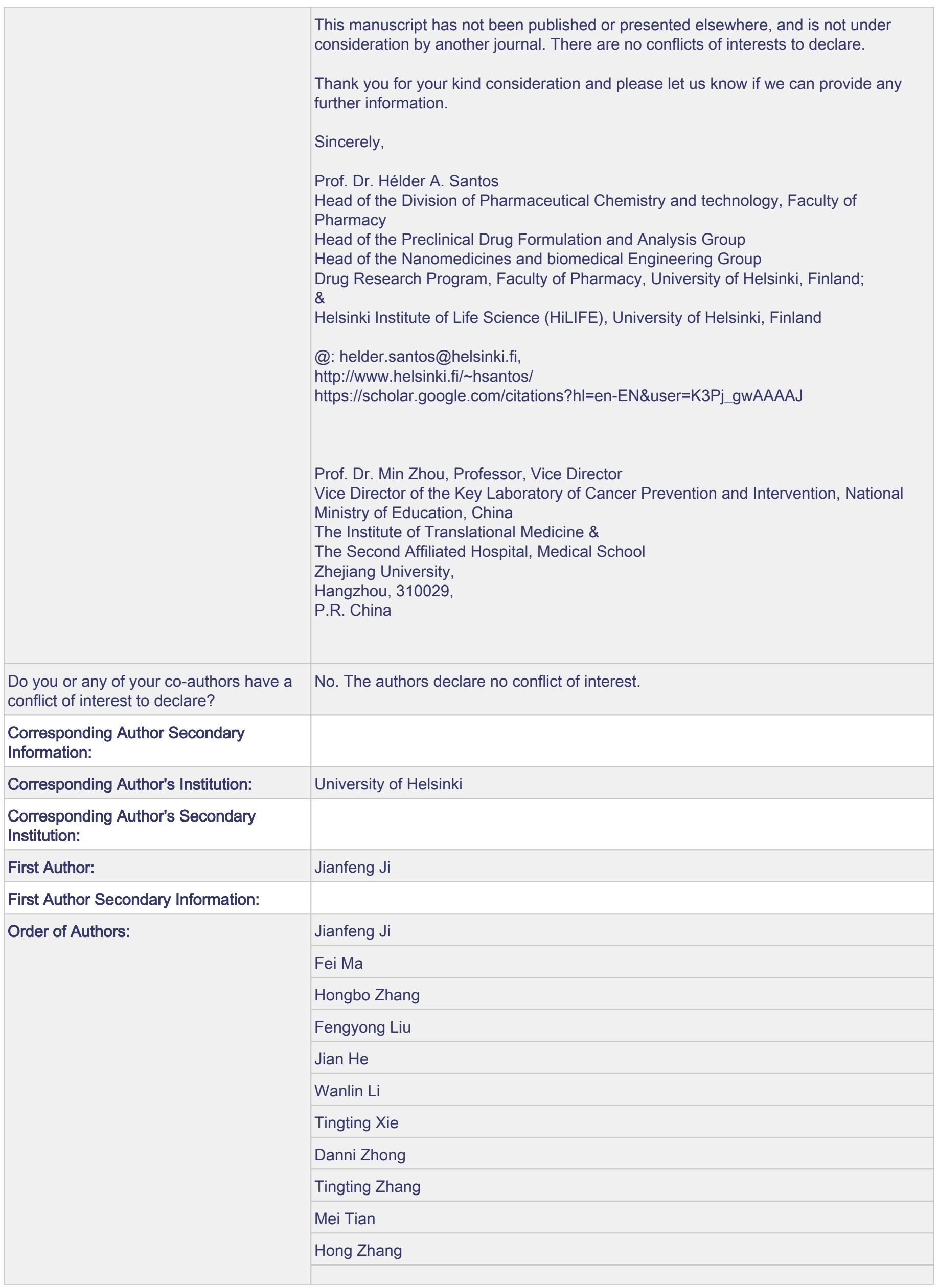


Helder Santos, D.Sc. (Chem. Eng.)

Min Zhou

Order of Authors Secondary Information:

$\begin{array}{ll}\text { Abstract: } & \text { Triple-negative breast cancer (TNBC) is a kind of aggressive malignancy with fast } \\ \text { metastatic behavior. Here, we developed a nanosystem loaded with a near-infrared } \\ \text { (NIR) agent to achieve chemo-photothermal combination therapy for inhibiting tumor } \\ \text { growth and metastasis in TNBC. The NIR agent of ultrasmall sized copper sulfide } \\ \text { nanodots with strong NIR light-absorbing capability, is entrapped into the doxorubicin- } \\ \text { contained temperature sensitive polymer-based nanosystem by a self-assembled } \\ \text { method. The temperature sensitive multifunctional nanosystem (TSNCs) can } \\ \text { significantly enhance the drug penetration depth and significantly kill the cancer cells } \\ \text { under the near-infrared laser irradiation. Importantly, we found the tumor penetrating } \\ \text { nanosystem combined with NIR laser irradiation can prevent lung and liver metastasis } \\ \text { via extermination of the cancer stem cells. The in vivo characteristics, evaluated by } \\ \text { photoacoustic imaging, positron emission tomography imaging, pharmacokinetics, and } \\ \text { biodistribution, confirmed their feasibility for tumor treatment owing to their long blood } \\ \text { circulation time and high tumor uptake. Thanks to the high tumor uptake and highly } \\ \text { potent antitumor efficacy, the doxorubicin-induced cardiotoxicity can be avoided when } \\ \text { the TSNCs was used. Taken together, we believed that the nanosystem has excellent } \\ \text { potential for clinical translation. }\end{array}$




\section{WILEY-VCH}

DOI: 10.1002/ ((adfm.201801738))

Article type: Full Paper

\section{Light-Activatable Assembled Nanoparticles to Improve Tumor-Penetration and Eradicate Metastasis in Triple Negative Breast Cancer}

Jianfeng Ji, Fei Ma, Hongbo Zhang, Fengyong Liu, Jian He, Wanlin Li, Tingting Xie, Danni Zhong, Tingting Zhang, Mei Tian, * Hong Zhang, * Hélder A. Santos, * and Min Zhou*

J. Ji, F. Ma, J. He, T. Zhang, Prof. H. Zhang, Prof. M. Tian, Prof. M. Zhou

Department of Nuclear Medicine, The Second Affiliated Hospital, School of Medicine, Zhejiang University, Hangzhou 310009, P. R. China

E-mail: zhoum@zju.edu.cn; meitian@zju.edu.cn; hzhang21@ zju.edu.cn

F. Ma, W. Li, T. Xie, D. Zhong, Prof. M. Zhou

Institute of Translational Medicine, Zhejiang University, Hangzhou 310009, P. R. China

Prof. H. Zhang

Turku Center for Biotechnology, Åbo Akademi University, Turku, 20520, Finland

Prof. Hélder A. Santos

Drug Research Program, Division of Pharmaceutical Chemistry and Technology, Faculty of Pharmacy, University of Helsinki, FI-00014, Helsinki, Finland

E-mail: helder.santos@helsinki.fi

Prof. Hélder A. Santos

Helsinki Institute of Life Science, HiLIFE, University of Helsinki, FI-00014 Helsinki, Finland

Prof. F. Liu

Department of Interventional Radiology, Chinese PLA General Hospital, Beijing 100853, China

Prof. H. Zhang

Shanxi Medical University, Taiyuan, 030001

Prof. M. Zhou

Key Laboratory of Cancer Prevention and Intervention, National Ministry of Education, Zhejiang University, Zhejiang University, Hangzhou 310009, P. R. China

Prof. M. Zhou

State Key Laboratory of Modern Optical Instrumentations, Zhejiang University, Hangzhou 310058, P. R. China

J.J., F.M, and H.Z. contributed equally to this work.

Keywords: triple-negative breast cancer, nanoparticles, self-assembly, tumor penetration, cancer metastasis 


\section{WILEY-VCH}

Triple-negative breast cancer (TNBC) is a kind of aggressive malignancy with fast metastatic behavior. Here, we developed a nanosystem loaded with a near-infrared (NIR) agent to achieve chemo-photothermal combination therapy for inhibiting tumor growth and metastasis in TNBC. The NIR agent of ultrasmall sized copper sulfide nanodots with strong NIR lightabsorbing capability, is entrapped into the doxorubicin-contained temperature sensitive polymer-based nanosystem by a self-assembled method. The temperature sensitive multifunctional nanosystem (TSNCs) can significantly enhance the drug penetration depth and significantly kill the cancer cells under the near-infrared laser irradiation. Importantly, it is plausible that the tumor penetrating nanosystem combined with NIR laser irradiation can prevent lung and liver metastasis via extermination of the cancer stem cells. The in vivo characteristics, evaluated by photoacoustic imaging, positron emission tomography imaging, pharmacokinetics, and biodistribution, confirmed their feasibility for tumor treatment owing to their long blood circulation time and high tumor uptake. Thanks to the high tumor uptake and highly potent antitumor efficacy, the doxorubicin-induced cardiotoxicity can be avoided when the TSNCs was used. Taken together, we believed that the nanosystem has excellent potential for clinical translation. 


\section{WILEY-VCH}

\section{Introduction}

Breast cancer ranks second among the most prevalent malignancies in the world. ${ }^{[1,2]}$ Triple-negative breast cancer (TNBC) consists of 10-20\% of breast cancer cases and is considered as the most aggressive subtype. ${ }^{[3,4]}$ Due to the absence or low expression of progesterone, Her2/Neu, and estrogen receptors, TNBC is typically heterogeneous and aggressive. The prognosis of $\mathrm{TNBC}$ is bad and treatment options are limited. ${ }^{[5-7]}$ Consistent with its aggressive nature, within five years after diagnosis, TNBC is often associated with high rates of lung and liver metastasis, despite adjuvant chemotherapy. ${ }^{[8-}$ 10] Therefore, it is highly demanded to develop efficient therapeutic platforms for TNBC. Commonly, che motherapy is preferential treatment in breast cancer patients after surgery, while doxorubicin (DOX) is one of the most used chemotherapeutic drugs. ${ }^{[11,12]}$ However, DOX mediated chemotherapy was restricted by several major issues, such as severe cardiotoxicity and poor tumor penetration depth, in clinical practice. ${ }^{[13-16]}$ Further more, DOX relative treatment cannot restrain the metastasis of TNBC and inhibit tumor recurrences. ${ }^{[17,18]}$ Therefore, a rational, complementing chemotherapeutic agent would reduce drug dosage to overcome cardiotoxicity, improve tumor perfusion to maintain intratumoral drug delivery, and prevent the tumor metastasis is necessary.

Nanoparticle-based drug delivery system has emerged as an advanced technology for spatiotemporally controlled codelivery of different anticancer drugs to the tumor sites through the enhanced permeability and retention (EPR) effect. ${ }^{[19-21]}$ However, poor tumor deep penetration of nanotherapeutics, owing to the non-uniformly leaky vasculature and a dense interstitial structure of tumor, lead to suboptimal therapeutic effect and severely hinder the clinic translation of this developments. ${ }^{[22]}$ Ultrasmall nanoparticles have the ability to distribute more homogeneity within tumors. ${ }^{[23]}$ It has been shown that $7 \mathrm{~nm}$ serum protein albumin nanoparticles have four times more apparent tissue permeability 


\section{WILEY-VCH}

than the large-sized nanoparticles in several tested breast cancer mouse models. ${ }^{[24]}$ Unfortunately, the ultrasmall sized nanoparticles (less than $7 \mathrm{~nm}$ ) limit their efficacy for tumor targeting due to their rapid clearance characteristics. ${ }^{[25-27]}$ On the contrary, larger nanoparticles with slower migration rate are more advantageous for high accumulation and retention in the tumor, but they are inherently unfavorable for reaching the deep region of the tumor due to their giant pervasion obstruction. ${ }^{[28]}$ Therefore, for high accumulation and deep tumor penetration, an ideal nanocarrier should be able to change to small size from its initial large size that favors longer circulation and selective extravasation. ${ }^{[24,29-36]}$ Previous studies have shown that nanoparticles in the range of 30 $50 \mathrm{~nm}$ exhibit great tumor uptake, excellent penetration capability for poorly permeable tumors to achieve the better antitumor effect. ${ }^{[37]}$

Here, we developed a novel nanosystem by a designed assembly method to overcome tumor penetration depth, tumor metastasis, and cardiotoxicity. We predicted that the large $(\sim 36 \mathrm{~nm})$ self-assembled temperature sensitive nanoclusters (TSNCs) could possess good blood persistence and preferential tumor accumulation due to their EPR effect. The TSNCs could be disassembled into ultrasmall nanodots $(\sim 6 \mathrm{~nm})$ under the near-infrared (NIR) laser irradiation, accelerating the deep penetration in the intratumoral environment. The pharmacokinetics, biodistribution, and tumor accumulation characteristics of the TSNCs were investigated by radio-labeling, and photoacoustic imaging. The laserinduced tumor penetration capability of the TSNCs was verified by tumor spheroids and tissue Immunol histochemistry. The antitumor efficacy and tumor metastasis were evaluated by tumor growth monitoring, tumor initial cells analysis, the histological analysis of triple-negative 4T1 breast tumor model. Moreover, the cardiotoxicity during the treatment was tested by ultrasound imaging. 


\section{WILEY-VCH}

\section{Results and Discussion}

\subsection{Synthesis and Characterization}

The copper sulfide nanodots (CuSNDs), DOX, and poly(lactic-co-glycolic acid (PLGA) were incorporated into the hydrophobic part of the F127 micelles using oil-in-water emulsion. Subsequently, the solvent was evaporated, and the emulsion was solidified to TSNCs. Transmission electron microscopy (TEM) analysis showed the TSNCs particle size is ca. 36 nm (Figure 1A,B). The inset image of Figure 1A showed that the nanostructure was composed of several ultrasmall sized nanoparticles. They were well-dispersed in water, and the hydrodynamic diameter evaluated by dynamic light scattering was found to be $\sim 40 \mathrm{~nm}$ (Figure S1A, Supporting Information), which is the preferable size for EPR effect for passive tumor targeting. The polydispersity index is 0.15 . The UV-vis-NIR spectra (Figure 1C) of TSNCs displayed a characteristic strong absorption peak at $980 \mathrm{~nm}$ and a small absorption peak at $540 \mathrm{~nm}$, corresponding for the component of CuSNDs and DOX in the TSNCs, respectively. Exposure of the TSNCs solution (2 OD, $100 \mu \mathrm{g} \mathrm{mL} \mathrm{m}^{-1}$ ) under continuous-wave NIR laser (808-nm) irradiation resulted in the raised solution temperature ranging from 20.1 to $87.8{ }^{\circ} \mathrm{C}$ with various power densities within a few minutes (Figure 1D). From the temperature change curves, the obvious heating effect was observed, which further validated that the efficacy of TSNCs as a photothermal coupling agent. Due to photothermal conversion, the NIR-induced heat destroyed the structure of TSNCs, leading to disassembly and release of the DOX and the ultrasmall sized CuSNDs $(\sim 5 \mathrm{~nm})$. The size distribution dramatically decreased from tens of nanometers to a few nanometers (Figure 1E,F), and the hydrodynamic size and polydispersity index of the disassembled nanoparticles measure were around $6.9 \mathrm{~nm}$ and 0.08, respectively (Figure S1B, Supporting Information). There was no significant change of particle size between before and after laser treatment, when no CuSNDs in the assembled nanoparticles (Figure S2, Supporting Information), indicated that the disassembly 


\section{WILEY-VCH}

of the TSNCs were attributed to the CuSNDs mediated photothermal effect. No particle aggregation was observed after 5 days of incubation in PBS solution at $37{ }^{\circ} \mathrm{C}$, and the hydrodynamic sizes of the TSNCs measured by DLS showed there are almost no differences at 1 or 5 days (Figure S3, Supporting Information), indicating the excellent physiological stability of TSNCs.
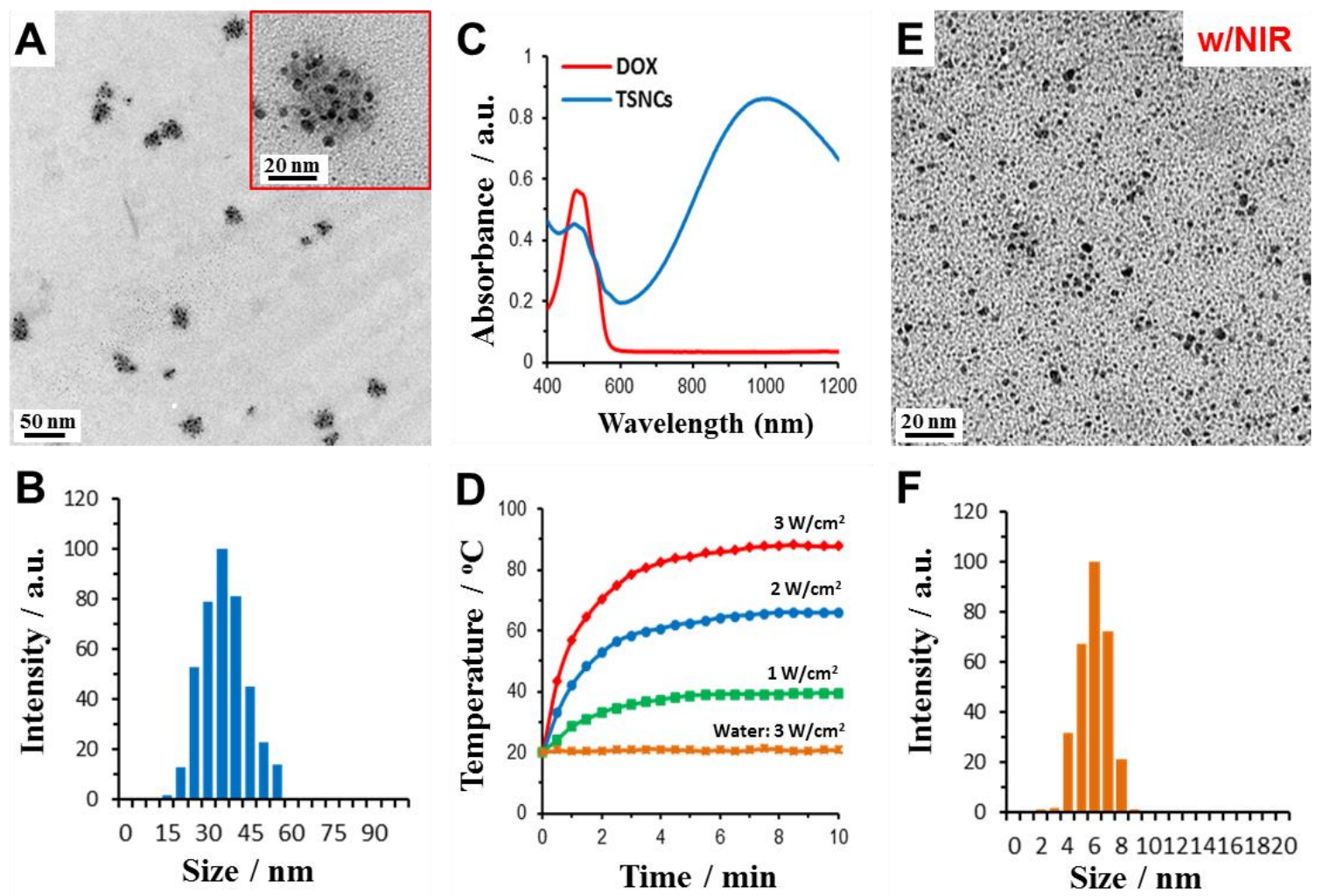

Figure 1. Characterization of TSNCs. (A) TEM images and (B) particle size distribution of TSNCs, inset: high-resolution images of TSNCs; (C) UV-vis-NIR spectra of TSNCs and DOX; (D) Temperature change curve of TSNCs under 808-nm laser irradiation with different laser power; (E) TEM images and (F) particle size distribution of TSNCs after laser irradiation.

\subsection{Dose- and Time-Dependent Cytotoxicity and Antitumor Efficacy In Vitro}

The DOX loading efficiency of the TSNCs was determined to be as high as $25.1 \%$ (by weight). We next investigated the drug release kinetics to check whether DOX could be efficiently released from TSNCs under the NIR laser. When TSNCs were incubated in PBS 


\section{WILEY-VCH}

without NIR laser exposure, the accumulated released DOX was extremely low $(<4 \%)$ over a period of 60 min (Figure S4, Supporting Information). However, when the TSNCs were irradiated by NIR laser, about $90 \%$ of DOX was quickly released from the TSNCs by the laser power of $2.0 \mathrm{~W} \mathrm{~cm}^{-2}$, suggesting NIR laser-triggered drug release as a result photothermal effect of CuSNDs from the TSNCs. Under the NIR laser irradiation, the temperature of the TSNCs can be elevated rapidly by the photothermal energy conversion of the CuSNDs. Thus, the constructed TSNCs was destroyed by the high temperature to promote DOX release from the cracked nanostructures.

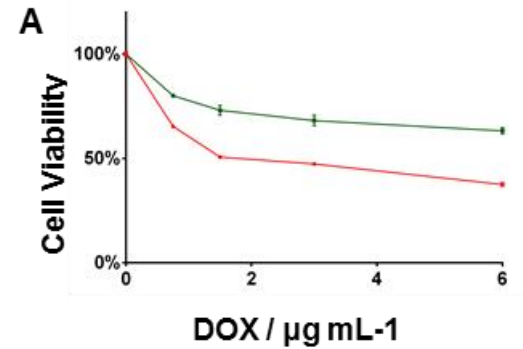

D

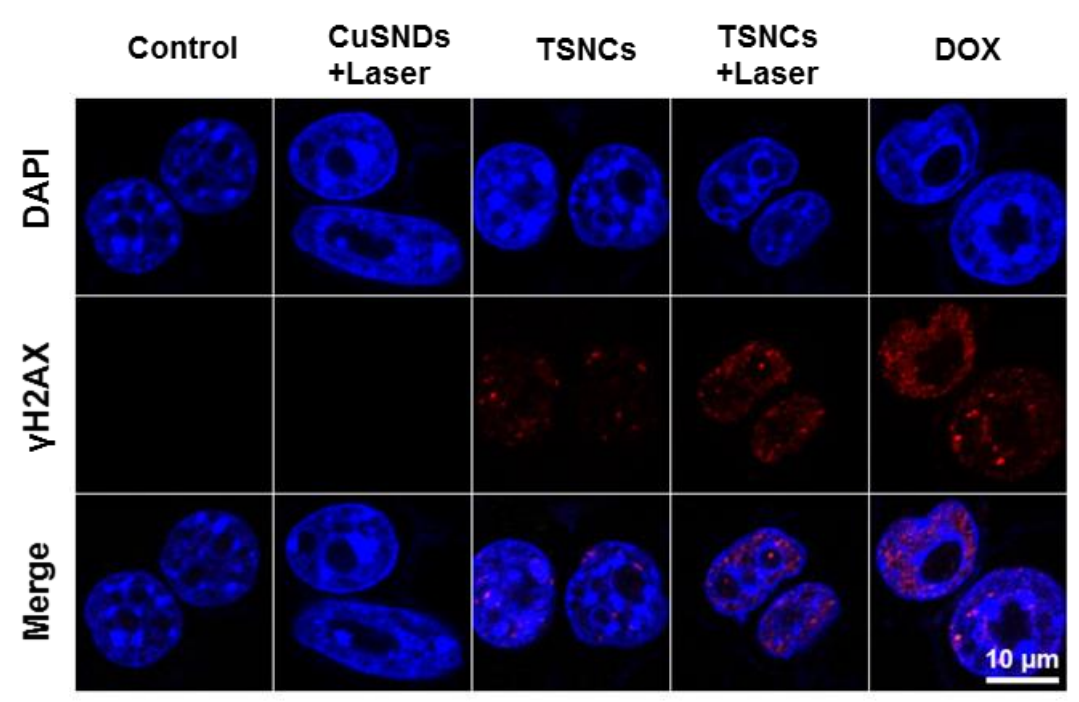

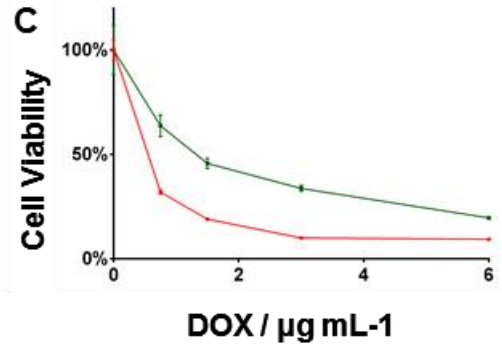

$\mathbf{E}$

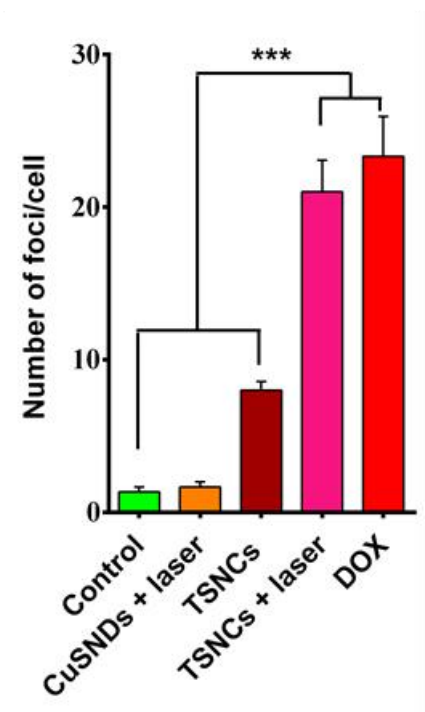

Figure 2. Cytotoxicity comparison of $4 \mathrm{~T} 1$ cells incubation with DOX or TSNCs for $24 \mathrm{~h}(\mathrm{~A})$, $48 \mathrm{~h}$ (B), and $72 \mathrm{~h}(\mathrm{C})$, respectively; (D) NIR laser-triggered DOX release for chemotherapy and its influence on cell DNA damage evaluated by $\gamma-\mathrm{H} 2 \mathrm{AX}$ foci; (E) Quantification of the Number of foci/cell. 


\section{WILEY-VCH}

DOX shows toxicity at the high concentration. We hypothesize the TSNCs have lower cytotoxicity owing to their lower DOX release characteristics than DOX. Therefore, dose- and time-dependent cytotoxicity of DOX and TSNCs were evaluated in 4T1 breast cancer cell by MTT assay (Figure 2A-C). The cytotoxicity of both DOX and TSNCs increased with the increase of the time and dose. TSNCs was obviously less toxic than free DOX, which demonstrated that only a limited part of DOX was released from TSNCs when there was no laser irradiation. We also evaluated the cell viability of the various treatment, including DOX, TSNCs, TSNCs+laser, CuSNDs, and CuSNDs+laser, with different concentrations at $24 \mathrm{~h}$ after treatments (Figure S5, Supporting Information). The TSNCs+laser treatment showed the lowest viability in all the treatment, demonstrating the best anticancer effect.

Next, cell DNA damage of DOX, TSNCs, and TSNCs plus laser (TSNCs+Laser) was studied by confocal fluorescent microscopy. Figure 2D showed that TSNCs+Laser treatment resulted in significant DNA damage $(\gamma-\mathrm{H} 2 \mathrm{AX}$ staining) than DOX and TSNCs treatment. Quantitative analysis (Figure 2E) indicated that around 10-fold or 3-fold higher red fluorescence $(\gamma-\mathrm{H} 2 \mathrm{AX}$ staining) were detected in TSNCs+Laser treated 4T1 cancer cells than CuSNDs+Laser or TSNCs treated groups. These results provided clear evidence that TSNCs induce stronger cell damage effect of $4 \mathrm{~T} 1$ cancer cells.

To compare the cellular uptakes of the nanoparticles with or without laser treatment, CuSNDs and TSNCs labeled with the NIR fluorescence dye Cy5.5, were incubated with 4T1 breast cancer cells. The strongest fluorescence signals from TSNCs+laser group were observed in the $4 \mathrm{~T} 1$ cells compared to CuSNDs, CuSNDs+Laser, and TSNCs group, indicating much more TSNCs in the cells after laser irradiation (Figure S6, Supporting Information). 


\section{WILEY-VCH}

\subsection{In Vitro Deep Tumor Penetration of DOX Induced by Laser Irradiation}

To find out whether the TSNCs+Laser might result in enhanced DOX penetration in tumor, we used the multicellular spheroids (MCSs) derived from 4T1 breast cancer cells as an in vitro model. The tumor spheroids were treated with free DOX, TSNCs, and TSNCs+Laser for $24 \mathrm{~h}$, and the penetration process was measured by using confocal microscopy. As shown in Figure 3A, TSNCs irradiated by laser caused that more DOX was penetrated into the 4T1 3D tumor spheroids than free DOX and TSNCs. Fluorescence intensities of DOX were similar in $10 \mu \mathrm{m}$ depths from the bottom of the tumor spheroids in DOX, TSNCs, and TSNCs+Laser groups. When the depths come to $50 \mu \mathrm{m}$ and $100 \mu \mathrm{m}$, the DOX fluorescence intensities in TSNCs+Laser group were obviously higher than those in DOX and TSNCs groups, indicating their high tumor penetration capability.

We further evaluated anticancer efficacy against $4 \mathrm{~T} 1$ cancer cells by MTT in vitro. As expected (Figure 3B), no obvious inhibition was observed over the no-treatment control, laser treatment, and CuSNDs groups, while TSNCs, free DOX, and CuSNDs with laser treatment (CuSNDs+Laser), showed limit cytotoxicity to the 4T1 cells. Obviously, TSNCs with laser treatment (TSNCs+Laser) showed best anticancer effect. About $30 \%$ or $55 \%$ of cells were killed by TSNCs or free DOX due to its anticancer effect, respectively, and about $69 \%$ of cells were killed in the CuSND+Laser group owing to the photothermal effect. When the cells were treated by TSNCs+Laser, more than $80 \%$ of cells were killed, which indicated the chemo- and photothermal synergistic therapy. These results collectively confirmed that the TSNCs are potentially efficient anticancer agents for cancer treatment. 


\section{WILEY-VCH}
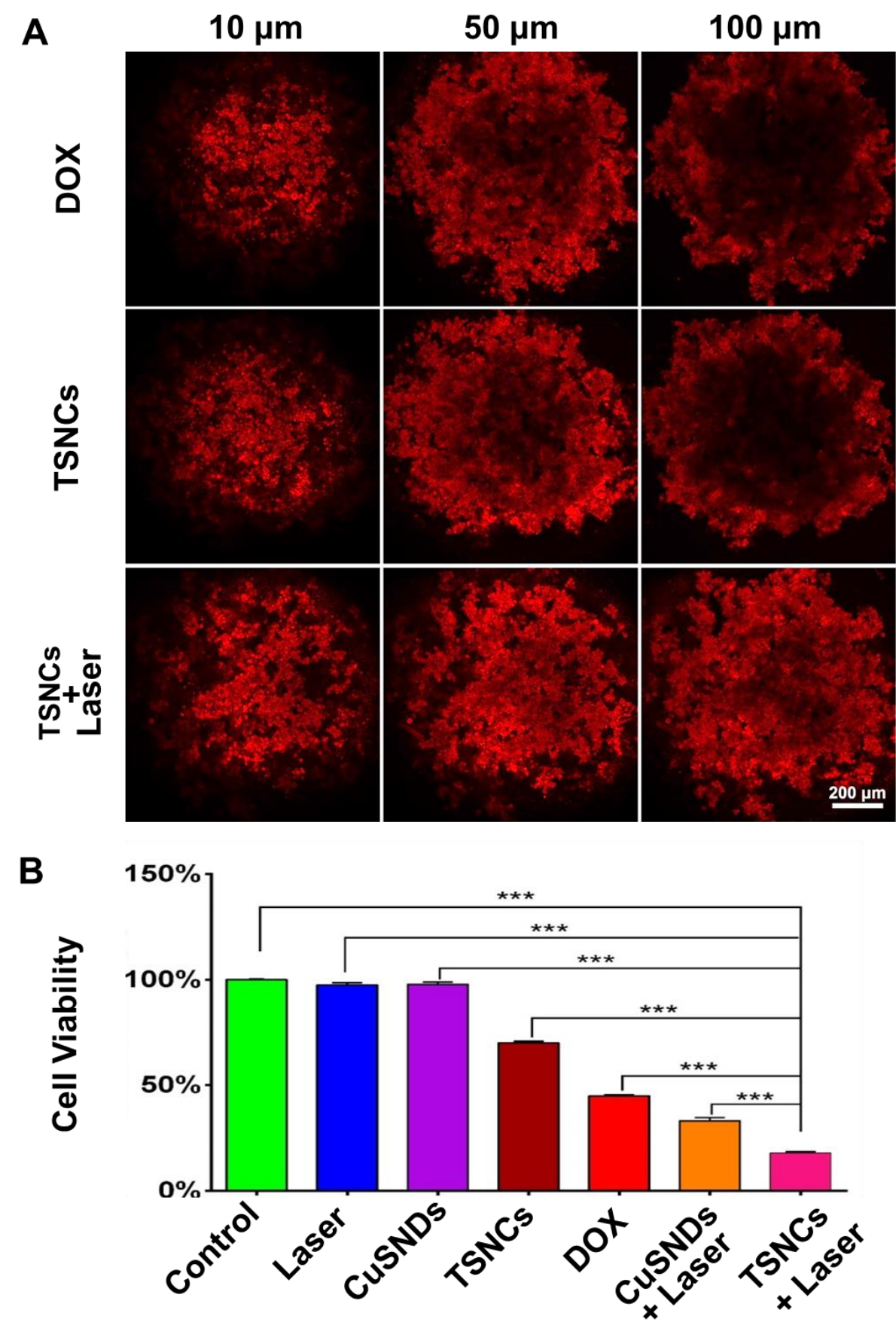

Figure 3. (A) Deep penetration of DOX/TSNCs in 4T1 3D tumor spheroids. Z-stack images using confocal microscopy of DOX penetration into 3D 4T1 tumor spheroids treated with free DOX or TSNCs (with or without NIR laser). Scale bar was $200 \mu \mathrm{m}$; (B) Cell viability of 4T1 breast cancer cells treated with PBS (control), laser only, CuSNDs, TSNCs, DOX, CuSNDs plus laser (CuSNDs+Laser), and TSNCs+Laser, respectively. After incubation of PBS (control), CuSNDs, DOX, TSNCs, then the cells in CuSNDs and TSNCs groups were irradiated at $808-\mathrm{nm}$ by NIR laser for $5 \mathrm{~min}$. (*** $\mathrm{p}<0.001)$. 


\subsection{In Vivo Photoacoustic Imaging, Radiolabeling, Pharmacokinetics, Biodistribution,}

\section{and, Fluorescence Imaging}

Photoacoustic imaging is an emerging imaging modality combining the advantages of high contrast optical imaging with ultrasound imaging in deep tissues. ${ }^{[38,39]}$ Since the TSNCs have a high absorbance in the NIR region, photoacoustic imaging was used to monitor their accumulation in tumors at different time intervals. We next investigated the feasibility of using TSNCs for in vivo tumor photoacoustic imaging. Figure $\mathbf{4 A}$ shows representative photoacoustic images of a mouse with a $4 \mathrm{~T} 1$ breast tumor, which are acquired at 1, 6, and 24 $\mathrm{h}$ after intravenous administration of TSNCs. As expected, TSNCs initially accumulated in the tumor tissue, allowing tumor visualization at $1 \mathrm{~h}$ after i.v. injection. Then, the photoacoustic intensity increased at $6 \mathrm{~h}$ and reached a higher level at $24 \mathrm{~h}$ post-injection. These results demonstrated that the constructed TSNCs showed substantial tumor homing ability, probably due to the EPR effect. In vivo photoacoustic imaging of CuSNDs in breast orthotopic $4 \mathrm{~T} 1$ tumor at different times after intravenous injection was also investigated. The contrast in the tumor (Figure S7, Supporting Information) was much weaker than TSNCs (Figure 4A), and no obvious enhancement was found after the time increased.

The radiolabeling technique was used extensively to assess the in vivo characteristics of nanoparticles due to its high sensitivity. ${ }^{[40-42]}$ We labeled TSNCs with radioisotope ${ }^{64} \mathrm{Cu}$ to investigate the pharmacokinetics, and biodistribution in vivo. The pharmacokinetic profile of the ${ }^{64} \mathrm{Cu}$-labeled TSNCs $\left(\left[{ }^{64} \mathrm{Cu}\right] \mathrm{TSNCs}\right)$ was measured by gamma counter to evaluate their time dependent blood concentrations. As shown in Figure 4B, the blood circulation of the TSNCs well conforms the two-compartment model. After the first distribution phase (rapid decline, half-life: $0.25 \pm 0.12 \mathrm{~h}$ ), the TSNCs had a long second elimination phase in circulating blood with a half-life of $7.20 \pm 0.35 \mathrm{~h}$, which is considered as the major process for TSNCs clearance. Nanoparticles with suitable modification such as poly(ethylene glycol) (PEG) or PLGA molecules usually exhibited related long blood circulation time. In this study, 


\section{WILEY-VCH}

PLGA was used during the synthesis. The introducing of PLGA may plan an important role to

enhance the circulation time of TSNCs in vivo. The long circulation of the TSNCs detains the macrophage clearance in the mononuclear phagocytic system (MPS), which may facilitate the tumor accumulation TSNCs by EPR effect.

A $\quad 1 \mathrm{~h}$

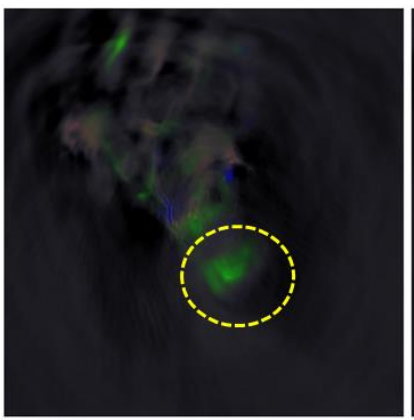

B

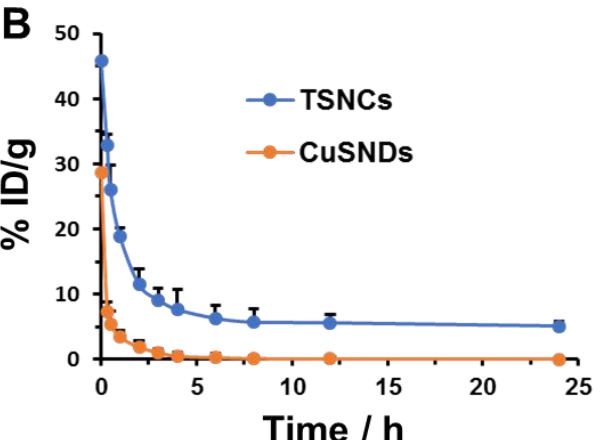

D

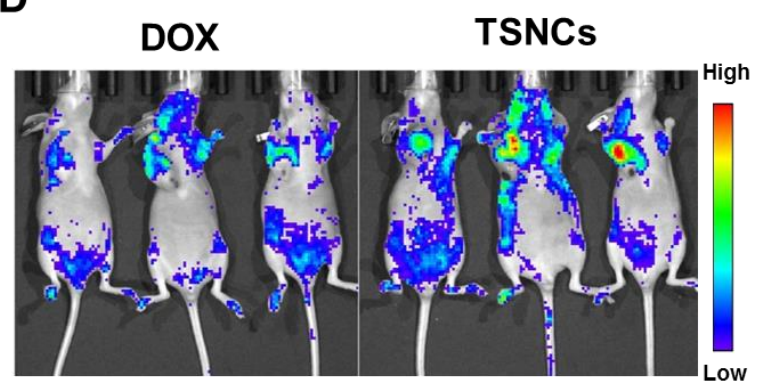

$\mathbf{E}$

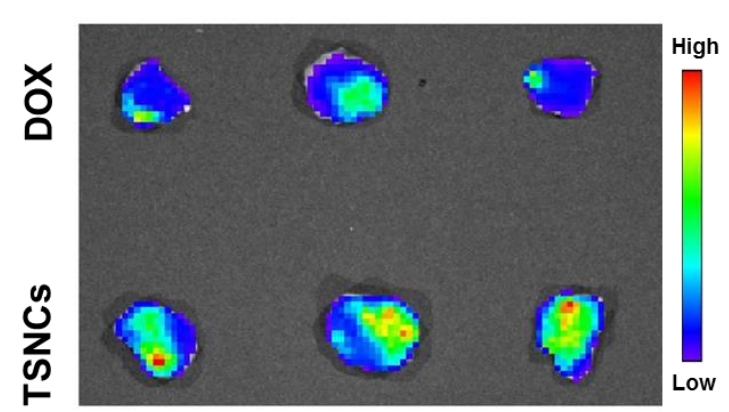

$24 \mathrm{~h}$
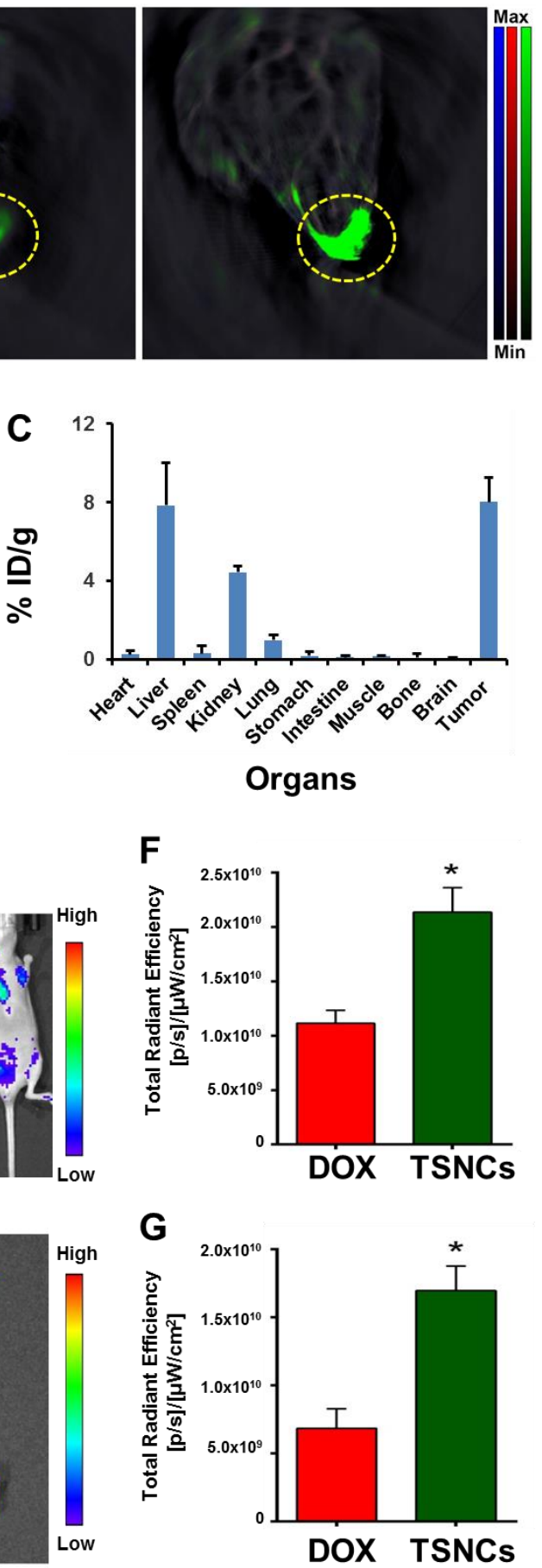

Figure 4. In vivo behaviors of TSNCs in breast orthotopic 4T1 tumor after intravenous injection. (A) Photoacoustic imaging of TSNCs at different time, the contrast in the tumor 


\section{WILEY-VCH}

(yellow dashed circle) was selectively enhanced; (B) Pharmacokinetics of TSNCs in mice from 0 to $24 \mathrm{~h}$ after intravenous injection $(\mathrm{n}=5)$; (C) Biodistribution of TSNCs $24 \mathrm{~h}$ postinjection ( $n=5)$; Fluorescence imaging of DOX/TSNCs in $4 \mathrm{T1}$ tumor beard mice (D) and the dissected tumors (E) by IVIS imaging ( $n=3$ ); Quantification of the mean fluorescent intensity of DOX/TSNCs in 4T1 tumor beard mice (F) and the dissected tumors (G).

We also investigated the biodistribution of radiolabeled $\left[{ }^{64} \mathrm{Cu}\right]$ TSNCs. Quantitative biodistribution analysis (Figure 4C) showed high tumor uptake of the TSNCs $\left(8.02 \% \mathrm{ID} \mathrm{g}^{-1}\right.$ ) at $24 \mathrm{~h}$ after i.v. injection, which was two-fold higher than CuSNDs (Figure S8, tumor uptake: $\left.3.27 \% \mathrm{ID} \mathrm{g}^{-1}\right)$. Notably, the efficient tumor uptake of the TSNCs should be attributed to their long circulation time in the blood (Figure 4B). The high level of liver uptake of the TSNCs is possibly owing to MPS absorption, whereas the uptake in the kidney can be possibly correlated with renal excretion.

Mice bearing 4T1 tumors were administered intravenously with DOX or TSNCs. In vivo imaging system (IVIS) optical imaging was carried out at $24 \mathrm{~h}$ after i.v. injection to observe the accumulation of DOX in the tumors. A significantly enhanced DOX fluorescence signal was detected in the tumors in vivo and ex vivo of $4 \mathrm{~T} 1$ tumors bearing mice treated with TSNCs than with DOX (Figure 4D,E). The quantitative analysis (Figure 4F,G) of the fluorescent intensity in TSNCs accumulated tumors at $24 \mathrm{~h}$ was significantly higher than in the DOX accumulated tumor $(\mathrm{p}<0.05)$. Ex vivo dissected tumor fluorescence imaging and quantitative analysis at $24 \mathrm{~h}$ showed the similar results. Ex vivo images (Figure 5A) showed low accumulation in major organs, including heart, liver, spleen, kidney, and spleen. Importantly, we found that the DOX accumulation in the heart of the TSNCs treated mice was much lower than the DOX-treated mice. The staining (DAPI) tissue slices (Figure 5B) and quantitative fluorescent signal analysis (Figure 5C) demonstrated low amounts of DOX in heart, liver, spleen, lung, and kidney tissues of TSNCs group, compared to DOX group. Those 


\section{WILEY-VCH}

data indicate possible lower cardiotoxicity of TSNCs. These results were consistent with the quantitative analysis of biodistribution.

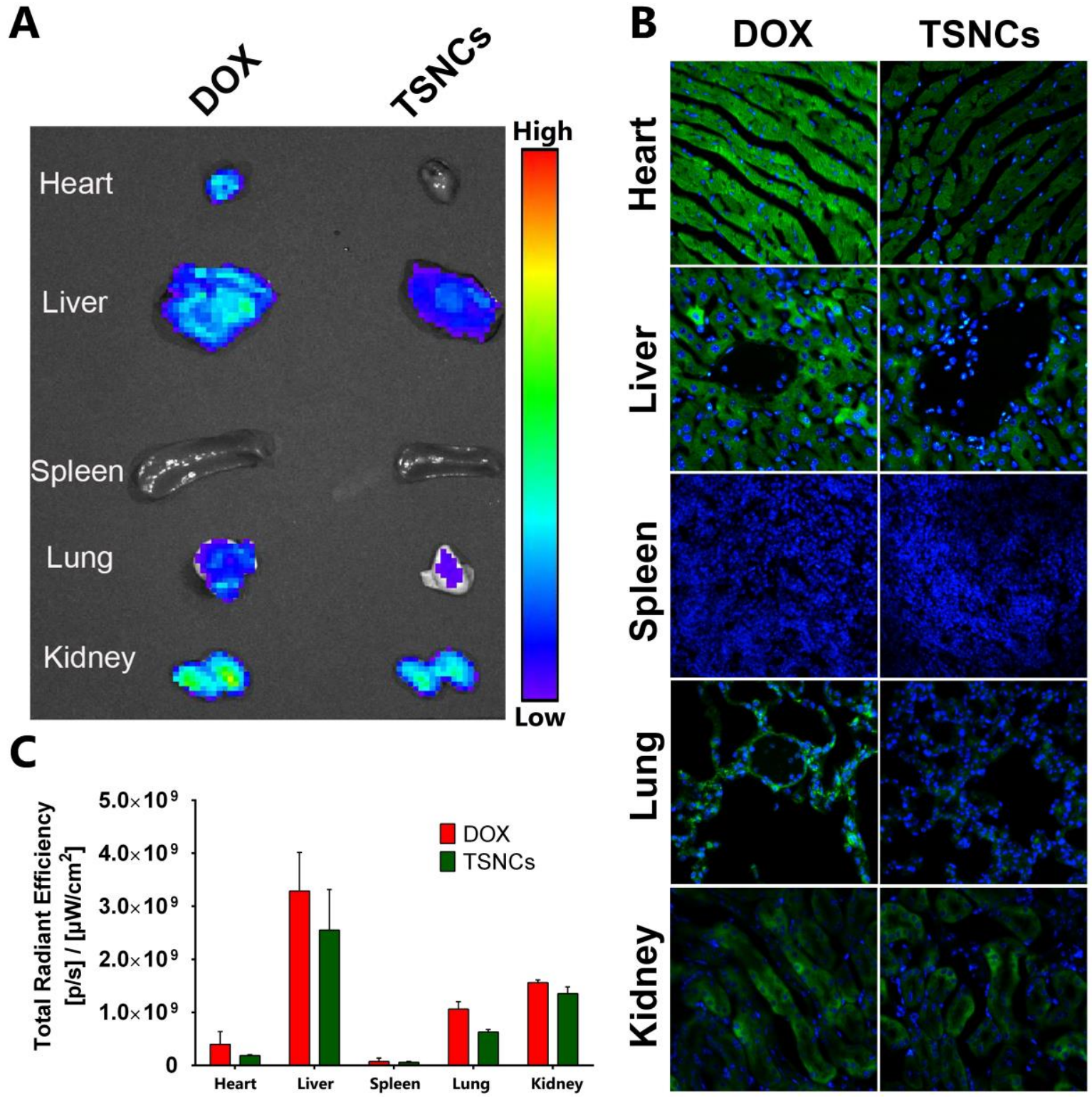

Figure 5. (A) Fluorescence imaging of major organs, including heart, liver, spleen, kidney, and lung after i.v. injection of DOX or TSNCs. (B) The tissues slices stained with DAPI (blue) showed DOX distribution in different major organs. (C) Quantification of the mean fluorescent intensity of DOX/TSNCs in major organs.

\subsection{In Vivo Deep Tumor Penetration of DOX Induced by Laser Irradiation}

Following the promising in tumor spheroids findings, the deep tumor penetration effects of DOX caused by laser irradiation were further investigated in solid tumors in vivo. Tumor 


\section{WILEY-VCH}

slices were stained with anti-CD34 and anti-HIF-1 $\alpha$ antibody to identify tumor blood vessels

and tumor hypoxia areas. In the immunofluorescence staining study (Figure 6A), for TSNCs+Laser treatment, the green DOX fluorescence showed higher DOX fluorescence intensity in tumor sites, indicating strong uniform perfusion in the tumor interstitium. However, the DOX only or TSNCs with laser irradiation displayed weak signal in the tumors. The low signal of DOX was attributed to the low tumor uptake of the DOX, whereas, the weak signal of TSNCs in the tumor tissue was because of their large particle size. This suggests that TSNCs+Laser treatment can release DOX at tumor sites and the released DOX enables efficient extravasation and penetration into deep tumor space, whereas the penetration depth of DOX only or TSNCs without laser irradiation was limited. Moreover, the fluorescence intensity of DOX significantly increased in farther distance from the CD34identified tumor blood vessels and in HIF-1 $\alpha$-identified tumor hypoxia areas when tumors treated with TSNCs subjected to additional laser irradiation (Figure 6B). These results confirmed that the TSNCs+Laser had better ability to deliver the drug to the deeper tumor penetration depth. Therefore, much more cancer cells could be killed by the higher drug concentration in the whole tumor area. 


\section{WILEY-VCH}
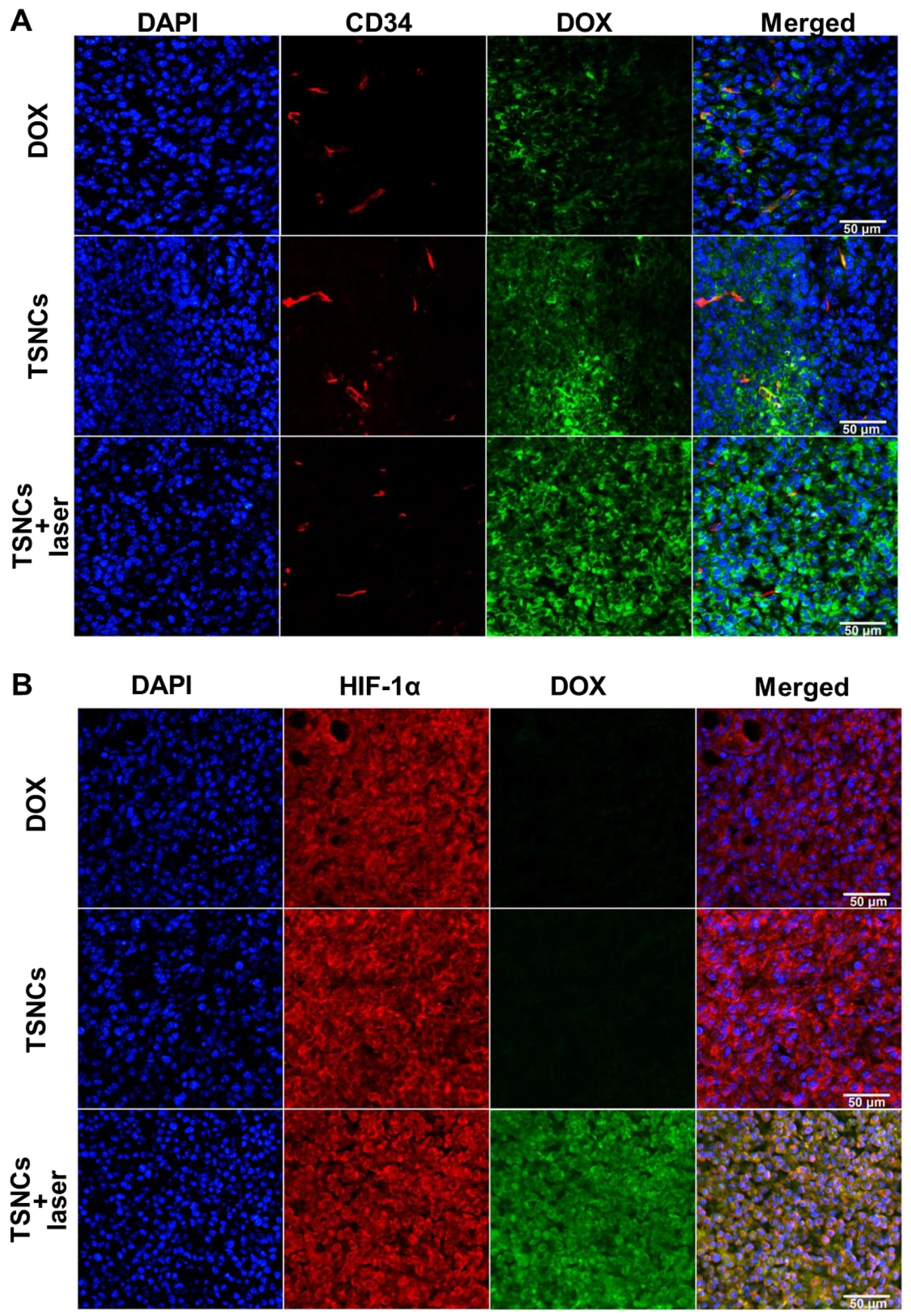

Figure 6. (A) DOX penetration into the vascular sparing region of tumors intravenously injected with free DOX or TSNCs (with or without NIR laser) using confocal microscopy; (B) DOX penetration into the Hypoxic region of tumors intravenously injected with free DOX or TSNCs (with or without NIR laser) using confocal microscopy. Scale bar was $50 \mu \mathrm{m}$. 


\section{WILEY-VCH}

\subsection{In Vivo Antitumor Efficacy}

Due to the excellently enhanced tumor accumulation of TSNCs and deep tumor penetration with laser irradiation, we rationally evaluated the antitumor efficacy in vivo in 4T1 tumors bearing mice. Tumor surface temperature monitored by using a thermal camera increased rapidly to about $50{ }^{\circ} \mathrm{C}$ in CuSNDs+Laser and TSNCs+Laser groups subjected to laser treatment while tumor surface temperature of laser group reached $40{ }^{\circ} \mathrm{C}$ (Figure $7 \mathbf{A , B}$ ). Before the tumor growth delay study, the alterations of cell apoptosis, cell proliferation, and cell thermal response in tumors were firstly identified by Hematoxylin and Eosin (H\&E), TUNEL, Ki-67, and HSP 70 staining assays (Figure 7C) shortly after a variety of treatments ( $4 \mathrm{~h}$ post-treatment). Owing to the poor tumor accumulation, the free DOX-induced limited apoptosis or proliferation inhibition of $4 \mathrm{~T} 1$ cells in vivo was found compared with that of the control group. Similarly, a small quantity of apoptosis and proliferation inhibition of 4T1 cells were observed in TSNCs group due to the poor DOX release and penetration in tumors. Compared with other groups, the treatment of TSNCs+Laser led to significantly more apoptosis and proliferation inhibition in the tumor. 
WILEY-VCH

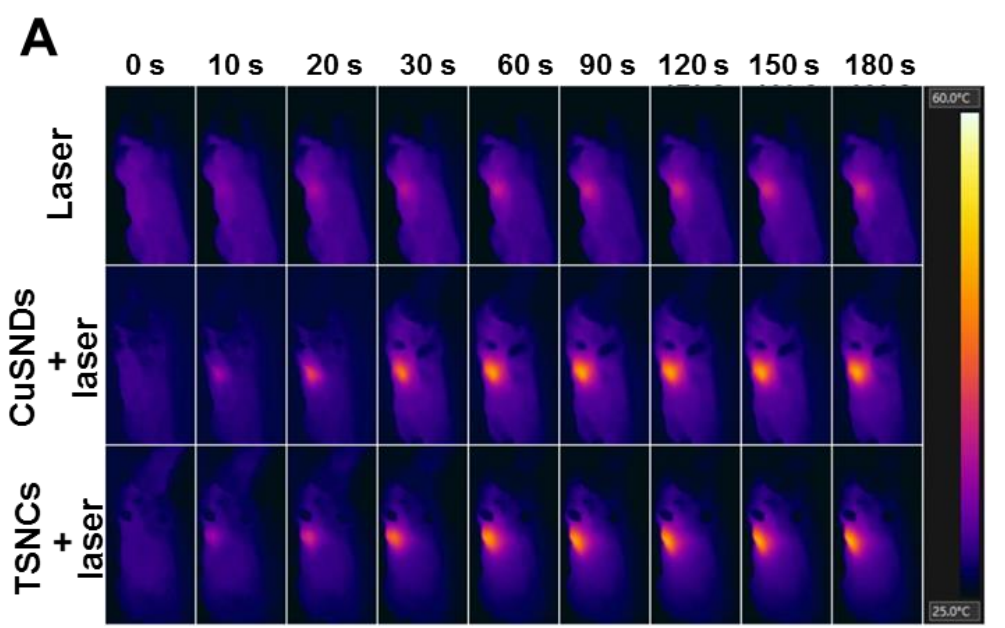

B
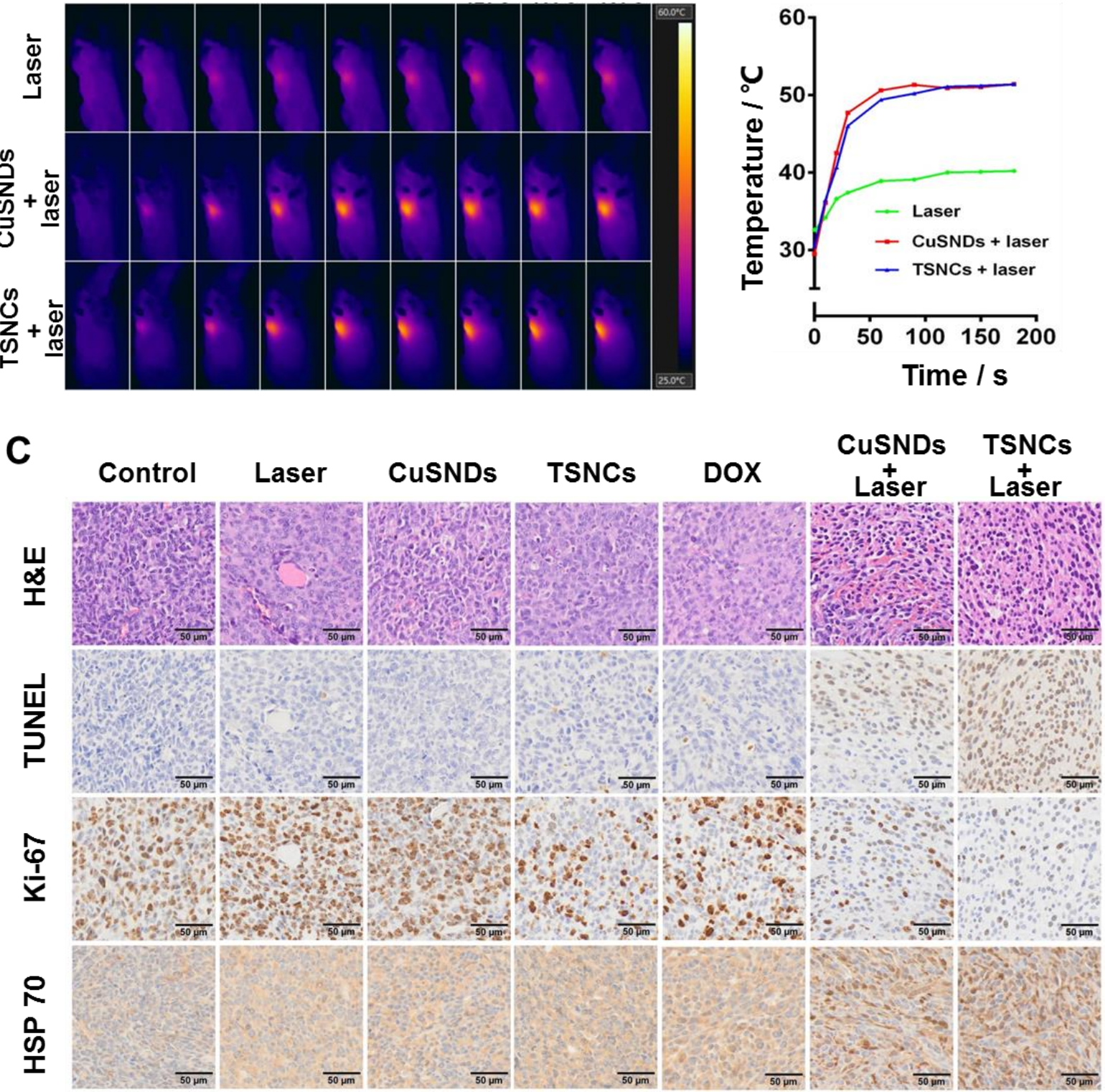

Figure 7. Short-term antitumor effect in vivo. (A) Infrared thermal of $4 \mathrm{~T} 1$ tumor bearing mice i.v. injected with saline, CuSNDs, TSNCs irradiated with NIR laser. (B) Temperature elevation at the tumor site after NIR laser radiation. (C) Representative H\&E, TUNEL, Ki-67, and HSP 70 staining of Tumor sections $4 \mathrm{~h}$ after various treatments.

In vivo tumor growth delay study was carried out on the $4 \mathrm{~T} 1$ ortho topic tumor model. Due to the EPR effect, the tumor volumes of TSNCs group mice were a little less than those of DOX group mice (Figure S9, Supporting Information),, but the antitumor efficacy was not satisfactory resulting from the poor penetration of the drug in the tumor (Figure $\mathbf{8 A}$ ). CuSNDs+Laser treatment could slightly decrease the tumor growth compared with control, 


\section{WILEY-VCH}

CuSNDs, DOX, and TSNCs group. As expected, TSNCs combined with laser irradiation with

passive tumor targeting efficiency and deep penetration of DOX in tumor inhibited the tumor growth more effectively than other treatments. By the end of the treatment procedure, the tumor-bearing mice were sacrificed, and the resected tumors were photographed and weighed. The average tumor weight of TSNCs+Laser group significantly reduced by $95.9 \%$ in comparison to the respective control group, suggesting the novel strategy was the most effective treatment in suppressing tumor growth (Figure 8B,C).

A
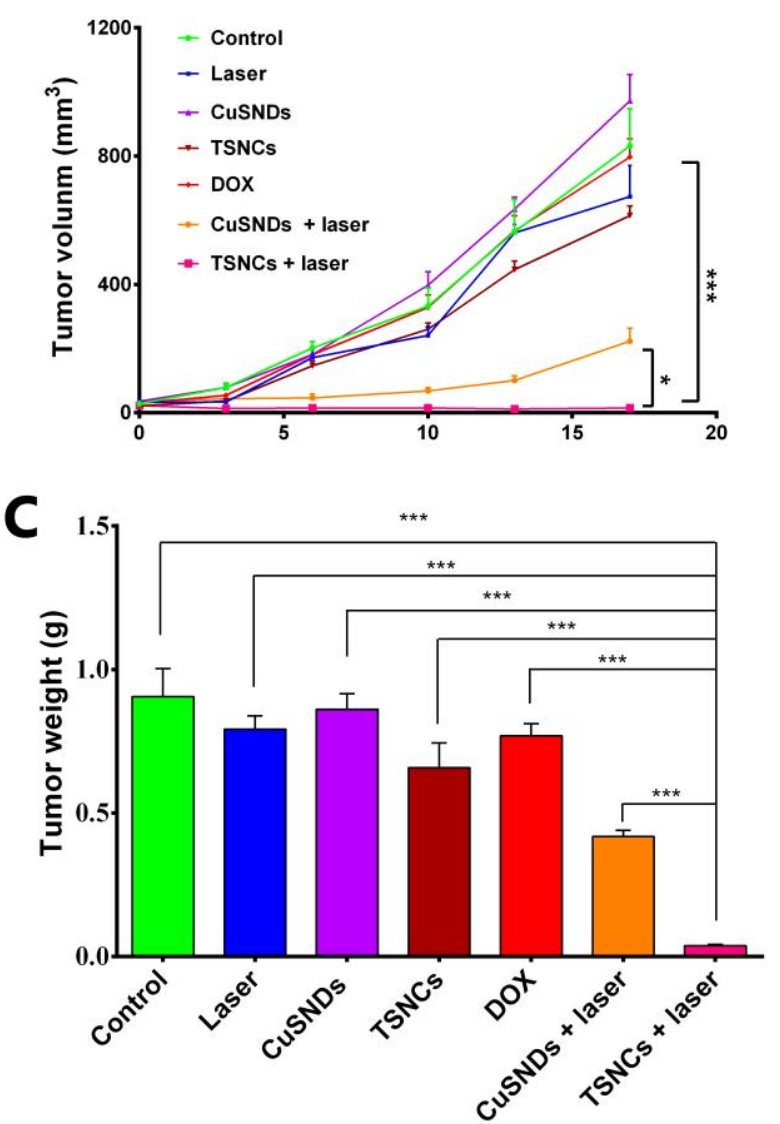

B

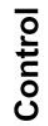

๖్

号

号
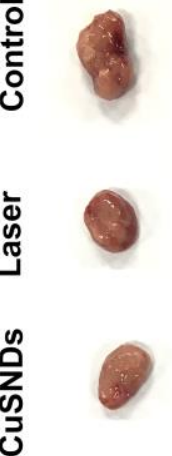

๖ั

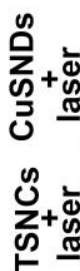

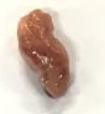
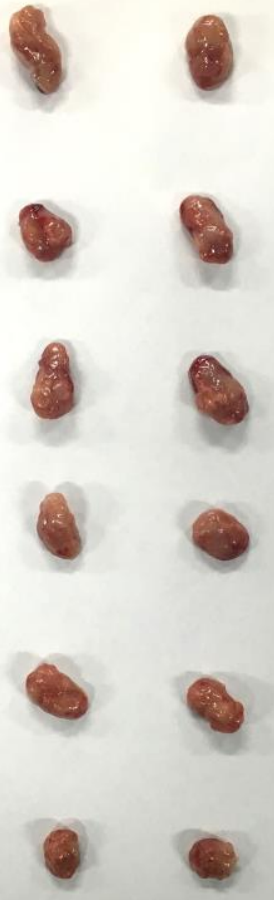

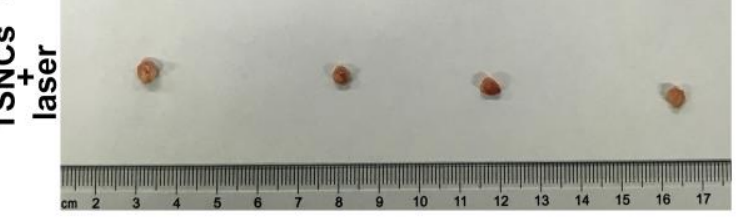

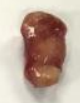
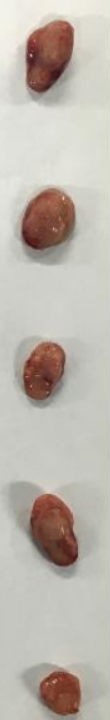

Figure 8. Antitumor effect in vivo. (A) Tumor growth curves of mice bearing 4T1 tumors followed by various therapeutic methods; (B) Representative photograph of dissected tumors; (C) Tumor weights of dissected tumors. $(* \mathrm{p}<0.05$, *** $\mathrm{p}<0.001)$ 


\section{WILEY-VCH}
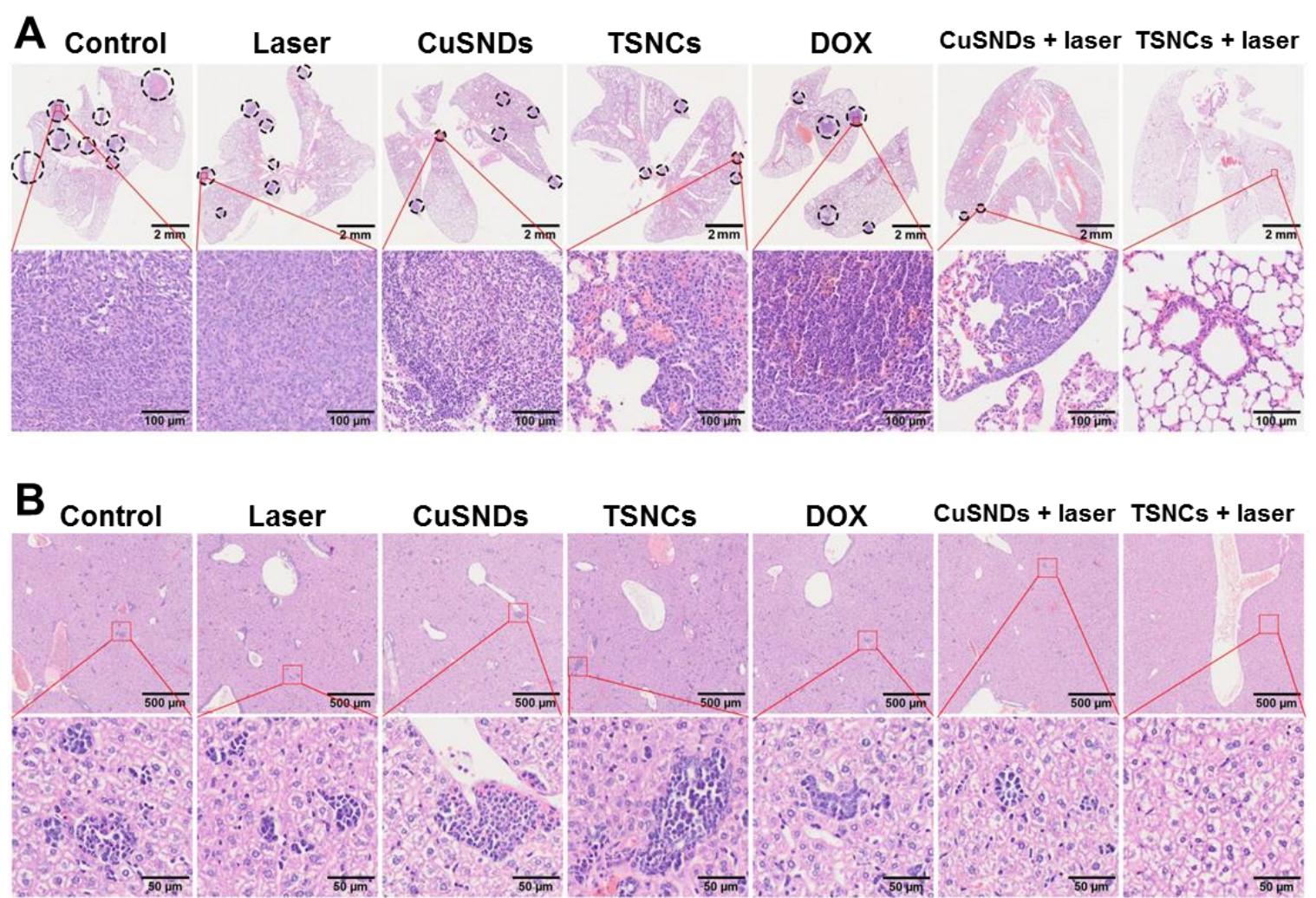

Figure 9. Anti-metastasis and tumor load-related response efficacy. (A) Representative photograph of pathological lung sections stained with H\&E from each group of mice; (B) Representative photograph of liver pathological sections stained with H\&E.

\subsection{Pulmonary and Hepatic Metastasis Evaluation}

Lethal pulmonary and hepatic metastasis is the main causes of mortality in breast cancer patients. ${ }^{[43,44]}$ In our study, we observed a large number of lung metastatic lesions in mice in the control, laser, CuSNDs, TSNCs, and DOX groups on day 17 (Figure 9A). Fewer metastatic lesions were observed in groups of mice treated with CuSNDs+Laser, indicating those treatments were not capable to prevent tumor spread. However, no visible lung metastatic lesion was observed for the mice treated with TSNCs+Laser. Quantitative analysis shows that the number of metastatic lung nodules was obviously reduced after TSNCs+Laser treatment. Hepatic micrometastases were also found in the livers of the mice in the control, laser, CuSNDs, TSNCs, DOX, and CuSNDs+Laser treatment groups. However, the hepatic micro-metastases were significantly reduced in the TSNCs+Laser-treated group. Hence, TSNCs+Laser could effectively inhibit distant metastasis of 4T1 cells. 
WILEY-VCH

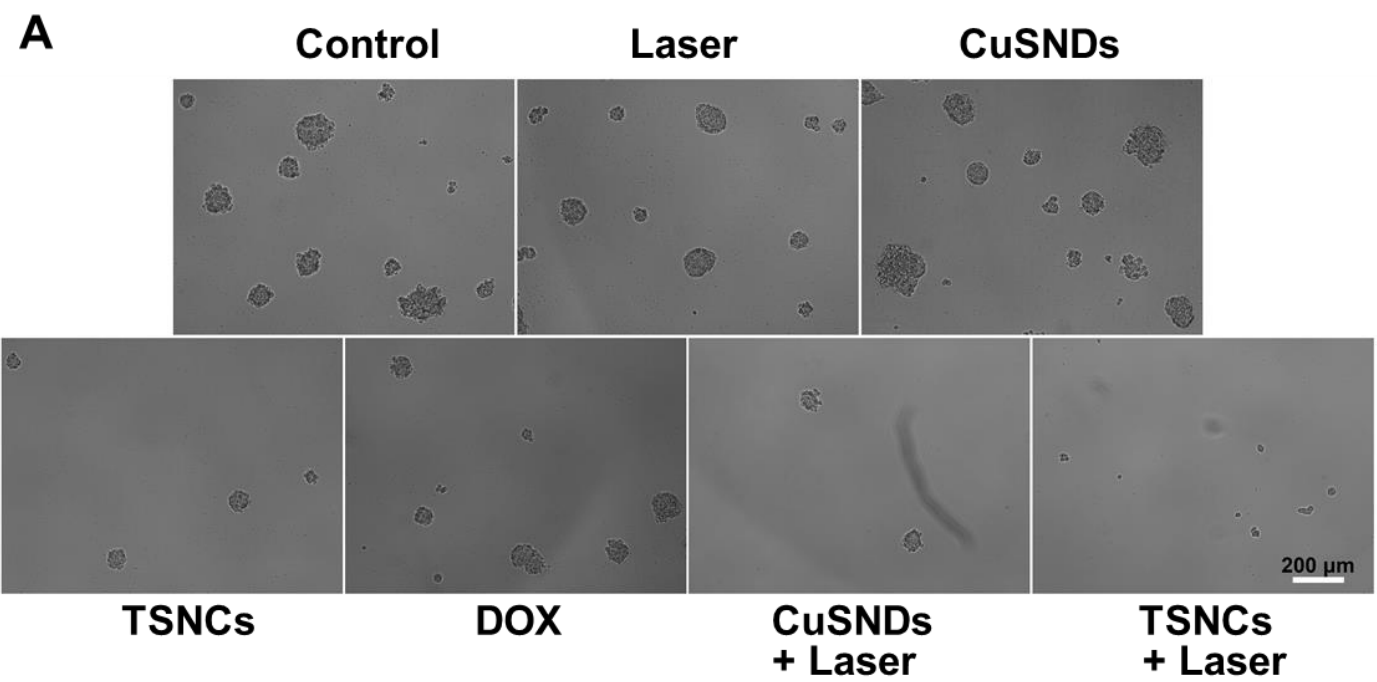

B

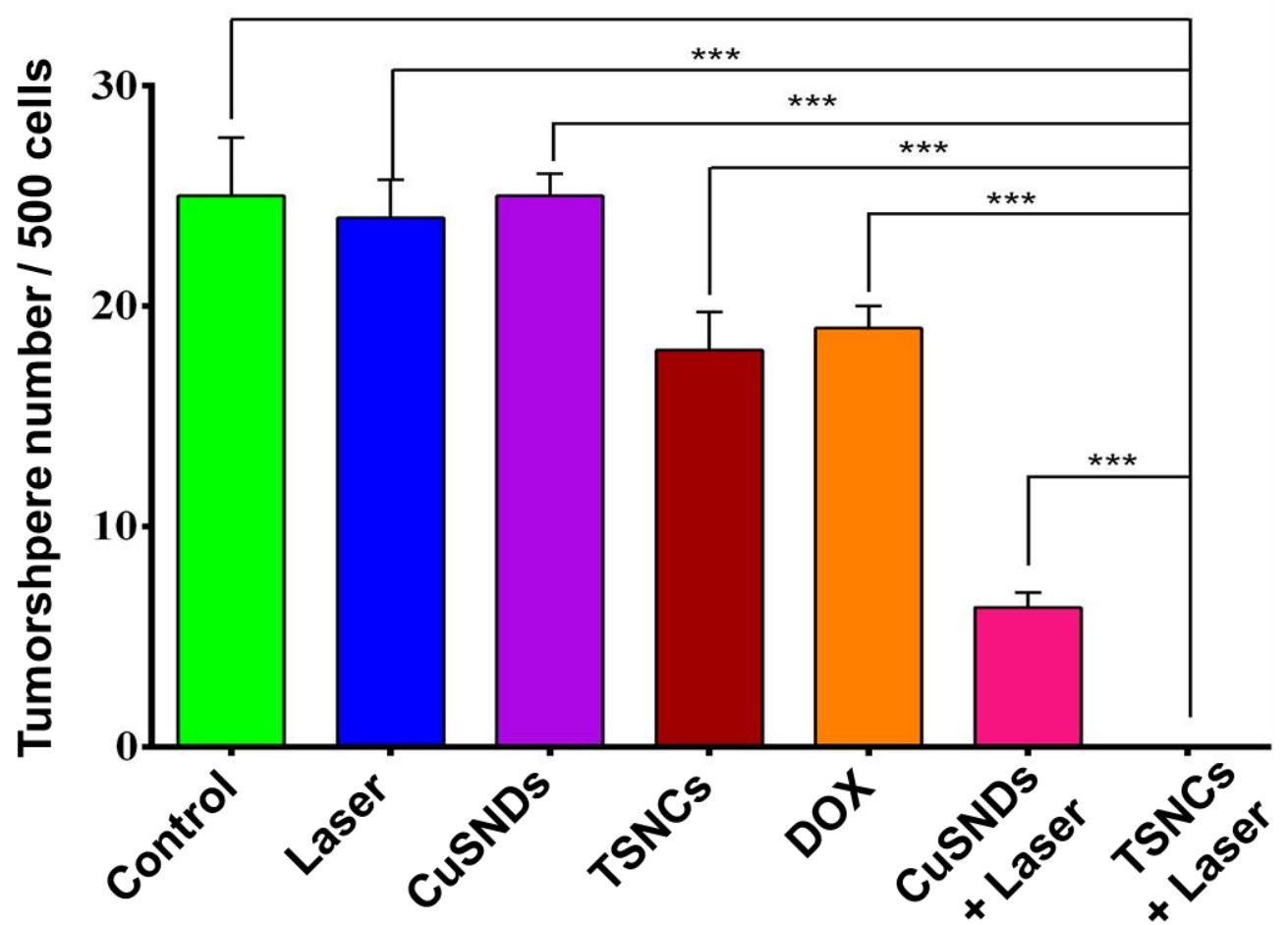

Figure 10. (A) Representative images of $4 \mathrm{~T} 1$ primary tumorsphere size seven days after various treatments; (B) 4T1 primary tumorsphere formation seven days after various treatments, grown at a density of 6000 cells/well. ***p $<0.001$.

\subsection{Eliminates cancer stem cells (CSCs).}

Breast CSCs are believed to the major reason of disseminated tumor metastases. ${ }^{[45-47]} \mathrm{We}$, therefore, determined whether TSNCs+Laser treatment could modulate CSCs populations in TNBC using tumorsphere assay. As shown in Figure 10, tumors treated with TSNCs+Laser exhibit most obviously decrease of tumorsphere formation. The quantitative analysis showed 


\section{WILEY-VCH}

a significant difference of tumorsphere number of the TSNCs+Laser group, compared with other treatment groups (Figure 10, ***p $<0.001$ ). These data suggest that TSNCs+Laser treatment enhance the killing effect against CSCs. The treatment efficiently eradicated breast CSCs and inhibited the lung and liver metastasis. TSNCs are promising candidates for cancer therapy. The development of lung metastasis is eventually happened for most breast cancer patients after treatment and accounts for about $60 \%-70 \%$ of death, which is possibly caused by CSCs due to their resistance to therapy. In our studies, DOX and CuSNDs moderately reduced the lung metastasis, and more importantly TSNCs+Laser treatment significantly reduced lung and liver metastas is in the murine 4T1 model. This is highly associated with the enhanced anti-CSC effect with TSNCs+Laser treatment, since the nanoparticles have long retention and deep penetration of the tumors. Moreover, the laser induced thermal-therapy also contributed to the CSCs killing. Therefore, the reduced metastasis of the mice in TSNCs+laser group could be attribute to the high-efficient CSCs killing effects and/or the reduced tumor burden by the combined therapeutic effects.

\subsection{In Vivo Cardiotoxicity Evaluation}

Despite DOX is an effective chemotherapeutic agent, cardiotoxicity is a major limiting factor in its use. Intravenously injection of TSNCs did not cause cardiotoxicity as evidenced by the echocardiography images. Figure $\mathbf{1 1}$ showed the comparison of the echocardiographic parameters at day 17 among the normal (control), DOX, and TSNCs+Laser groups. The animals in the DOX group had significantly reduced the ejection fraction (Figure 11A) and fractional shortening (Figure 11B) values on the day 17 when compared with the normal group. Also, the TSNCs+Laser group was found to similar parameters of cardiac function when compared to the DOX group. The representative images in each group are shown in Figure 11C. The left ventricles of the control and TSNCs+Laser treatment group were relatively narrow in comparison with the DOX-treated group. From the H\&E staining of heart 


\section{WILEY-VCH}

tissues, the DOX treated group showed a remarkable disorganization of cardiac muscle, edema and vacuolization of cells. However, there TSNCs+Laser group demonstrated the similar histological structures with no-treatment control group. This result demonstrates that TSNCs+Laser treatment can significantly reduce the cardiotoxicity induced by doxorubicin.

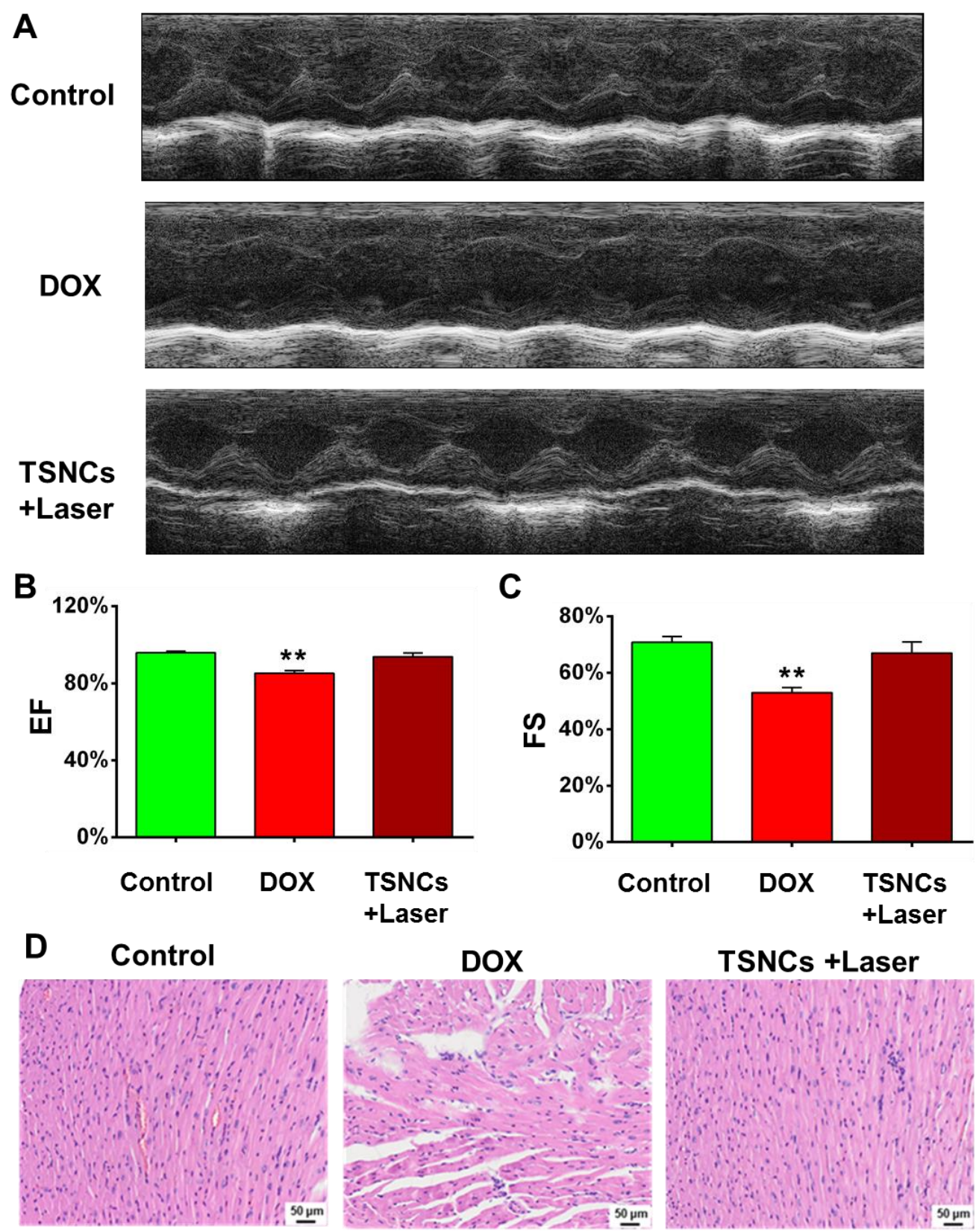

Figure 11. Changes in left ventricular function by control, DOX, or TSNCs+Laser treated mice. (A) Representative echocardiography images. (B) Percentage of ejection fraction (EF), and (C) fractional shortening (FS). ${ }^{*} \mathrm{p}<0.01$. (D) H\&E staining of heart tissues with various treatments. 


\section{WILEY-VCH}

\subsection{Preliminary Toxicity (Blood Sample Analysis)}

There was no difference in body weight changes during the whole treatment progress as shown in Figure S10A (Supporting Information). Consistent with the therapeutic effect, TSNCs combined with laser irradiation brought about the least lung and liver metastasis. All treatments in $4 \mathrm{~T} 1$ tumor-bearing mice didn't influence the normal range of red blood cell (RBC), platelet (PLT), hemoglobin (HB), alanine aminotransferase (ALT), aspartate transaminase (AST), creatinine (CREA), blood urea nitrogen (BUN) and the physiological morphology analyzed by H\&E stain (Figure S10B-S10H, Supporting Information). The preliminary investigations confirmed the TSNCs biosafety in vivo, indicating that TSNCs are vehicles for delivering DOX to effectively inhibit tumor growth with minimal toxicity at the tested dose. However, more systematic studies, such as long-term toxicity and immunecompatibility studies, are still necessary before the future clinical translation.

\section{Conclusion}

In summary, we have developed a novel tumor penetrating multifunctional nanosystem for the cancer combination therapy. The nanosystem has the following advantages over the previously reported nanostructure for cancer treatment. i) the nanosystem can significantly improve the drug penetration depth under NIR laser exposure due to their changeable particle size; ii) the nanosystem has neglect cardiotoxicity owing to their high tumor uptake; iii) the nanosystem can eradicate breast CSCs and reduced the tumor burden, and then could inhibit lung and liver metastasis of TNBC; i.v.) the nanosystem is non-toxic after intravenous administration. Such nanosystem with multiple therapeutic functions may have substantial potentials to clinical practice. 


\section{WILEY-VCH}

\section{Experimental Section}

Materials: All chemicals and reagents were used as received without any further purification. $\quad \mathrm{CuCl}_{2} \cdot 2 \mathrm{H}_{2} \mathrm{O}, \quad \mathrm{Na}_{2} \mathrm{~S}, \quad$ doxorubicin hydrochloride (DOX), $\mathrm{F}_{127}$ ((EO)97(PO)69(EO)97), and PLGA were purchased from Sigma-Aldrich (St. Louis, MO, USA). Trypsin-EDTA, dulbecco's modified eagle's medium (DMEM), and fetal bovine serum were obtained from Gibco-BRL (Burlington, ON, Canada).

Cell Culture and Animal Models: Mouse breast carcinoma 4T1 cell was obtained from the Institute of Biochemistry and Cell Biology (Shanghai, China). Cells cultured in DMEM supplemented with $10 \%$ FBS and $1 \%$ antibiotics $\left(100 \mathrm{U} \mathrm{mL}^{-1}\right.$ penicillin and $100 \mu \mathrm{g} \mathrm{mL}-1$ streptomycin) at $37^{\circ} \mathrm{C}$ and under a humidified atmosphere containing $5 \% \mathrm{CO}_{2}$. All animal experiments were approved by the Institutional Animal Care and Use Committee of Zhejiang University School of Medicine. Four- to six-weeks-old female BALB/c mice were used for the experiments. To establish orthotopic tumor model, $2 \times 10^{6} 4 \mathrm{~T} 1$ cells suspended in PBS were innoculated into the mammary fat pad of the mice.

Penetration of DOX/TSNCs (with or without laser) into Three-Dimensional (3D) Multicellular Tumor Spheroid :A density of 10,000 cells/well of 4T1 cells seeded onto microplates and incubated for $48 \mathrm{~h}$. After incubation of $10 \mu \mathrm{g} / \mathrm{mL}$ DOX, TSNCs with equivalent DOX concentration, cells of TSNCs with laser were then irradiated to NIR laser ( $2.0 \mathrm{~W} \mathrm{~cm}^{-2}, 3 \mathrm{~min}$ ). After another $4 \mathrm{~h}$ incubation, the spheroids were washed with PBS three times carefully, and then DOX fluorescence was measured using Z-stack imaging from the bottom of the spheroids with $10 \mu \mathrm{m}$ intervals by a confocal laser scanning imaging system (Nikon A1).

In Vivo and Ex Vivo Tumor Penetration of DOX/TSNCs (with or without laser): Tumorbearing (4T1) BALB/c mice were randomly divided into DOX, TSNCs and TSNCs+Laser groups ( $\mathrm{n}=3$, each group) by the tumor size reached around $5-8 \mathrm{~mm} .24 \mathrm{~h}$ after injected intravenously with DOX concentration of $5 \mathrm{mg} \mathrm{kg}^{-1}$ DOX or TSNCs, DOX and TSNCs groups of mice were photographed by IVIS Lumina LT Series III (Perkin Elmer). The two groups of mice were sacrificed after being observed, tumors and all major tissues, including heart, liver, spleen, lung, and kidney, were collected and photographed as well. The radiance of each photograph was analyzed by using Living Image 4.5 software (Perkin Elmer). $24 \mathrm{~h}$ after given TSNCs, the mice of TSNCs+Laser group were then exposed to 808-nm laser irradiation $\left(2.0 \mathrm{~W} \mathrm{~cm}^{-2}, 3 \mathrm{~min}\right)$ and were sacrificed $4 \mathrm{~h}$ later. 


\section{WILEY-VCH}

In Vivo Tumor and Tissue Distribution of DOX: Tumors and all major tissues of each

group above were fixed by paraformaldehyde. The equilibrated in $30 \%$ sucrose for an additional $24 \mathrm{~h}$. Sections of the tissues were cut at a thickness of $15 \mu \mathrm{m}$ on a freezing microtome. The tumor sections were incubated overnight with primary antibodies (rabbit monoclonal antibody against CD34 and rabbit monoclonal antibody against HIF-1 $\alpha$ ) after permeabilized with Triton X-100 and blocked by $10 \%$ normal calf serum. After washes with PBS, the sections were incubated with secondary fluorescent antibodies (Alexa Fluor 594 donkey anti-rabbit) for $1 \mathrm{~h}$. The 4',6-diamidino-2-phenylindole (DAPI) was used to stain the nuclei of both tumor sections and major tissues sections.

In Vivo Therapeutic Efficacy: When the average diameter of orthotopic 4T1 tumors reached about 5-8 mm, tumor-bearing female BALB /c mice were respectively randomly assigned to control, laser, CuSNDs, TSNCs, DOX, CuSNDs+Laser and TSNCs+Laser groups ( $n=3$, each group). Mice of control and laser groups were injected intravenously with saline. Mice of DOX were injected DOX intravenously at the dose of $5 \mathrm{mg} \mathrm{kg}^{-1}$. Mice of TSNCs and TSNCs+Laser groups were injected intravenously with TSNCs at the dose of $5 \mathrm{mg} \mathrm{\textrm {kg } ^ { - 1 }}$ (equivalent DOX concentration). Mice of CuSNDs and CuSNDs+Laser groups were injected intravenously with corresponding nanoparticles. $24 \mathrm{~h}$ later, the tumors in mice from the laser, CuSNDs+Laser, and TSNCs+Laser groups were irradiated with an 808-nm NIR laser (2.0 W $\mathrm{cm}^{-2}, 3 \mathrm{~min}$ ) and the temperature of the tumor was monitored by using a thermal camera (FLIR, ThermaCAM, U.S.A.). The mice of all the 7 groups were sacrificed $4 \mathrm{~h}$ after laser treatment, tumors were removed and placed in $4 \%$ paraformaldehyde for $24 \mathrm{~h}$, and then embedded in paraffin for analysis. Paraffin-embedded $5 \mu \mathrm{m}$ tumor sections were cut, deparaffinized, rehydrated. For Ki-67 and HSP 70 stain, slides were incubated overnight with rabbit monoclonal antibody against Ki-67 and rabbit monoclonal antibody against HSP 70 at $4{ }^{\circ} \mathrm{C}$ and treated with an appropriate secondary antibody for $1 \mathrm{~h}$ at room temperature. The DAB (3, 3'-diaminobenzidine tetrahydrochloride) liquid system was used to coloration. The TUNEL assay was performed to assess apoptosis by using the Apoptosis Detection Kit. After inactivated the endogenous peroxidase by $3 \% \mathrm{H}_{2} \mathrm{O}_{2}$, slides were incubated with the reaction mixture in a $37{ }^{\circ} \mathrm{C}$ humidified chamber for $60 \mathrm{~min}$ and incubated with anti-FITC HRP conjugate at $37{ }^{\circ} \mathrm{C}$ for $30 \mathrm{~min}$. After colored with $\mathrm{DAB}$ at room temperature for $10 \mathrm{~min}$, the reaction was terminated by washing with distilled water. The sections were observed with an Olympus VS120 slide scanner.

The tumor growth delay study was carried in both 4T1 tumor-bearing mice. When the tumors reached about $30 \mathrm{~mm}^{3}$, mice were randomly allocated into 7 groups $(\mathrm{n}=7)$ : control, 


\section{WILEY-VCH}

laser, CuSNDs, TSNCs, DOX, CuSNDs+Laser and TSNCs+Laser. Mice of control and laser groups were injected intravenously with saline. Mice of DOX were injected DOX intravenously at the dose of $5 \mathrm{mg} \mathrm{kg}^{-1}$. Mice of TSNCs and TSNCs+Laser groups were injected intravenously with TSNCs at the dose of $5 \mathrm{mg} \mathrm{kg}^{-1}$ (equivalent DOX concentration). Mice of CuSNDs and CuSNDs+Laser groups were injected intravenously with corresponding CuSNDs. The tumors in mice from the laser, CuSNDs+Laser, and TSNCs+Laser groups were irradiated with an 808-nm NIR laser $\left(2.0 \mathrm{~W} \mathrm{~cm}^{-1}, 3 \mathrm{~min}\right) 24 \mathrm{~h}$ later and a thermal camera (FLIR, ThermaCAM) was used to monitor the temperature of the tumor. After treatments, the length and width of the tumors were measured with a digital caliper every 2-3 day, and the tumor volumes were calculated as volume $\left(\mathrm{mm}^{3}\right)=$ length $\times$ width $\times 0.5$. Body weight of the mice was also recorded at the time of tumor size measurement. The $4 \mathrm{~T} 1$ tumor beard mice were sacrificed on day 17 . The blood of the mice was collected for blood routine, alanine aminotransferase (ALT), aspartate transaminase (AST), blood urea nitrogen (BUN) and creatinine (CREA) examinations. The tumors were collected, weighed, and the major organs were harvested and used for H\&E staining. The sections were obtained with an Olympus VS120 slide scanner.

\section{Supporting Information}

Supporting Information is available from the Wiley Online Library or from the author.

\section{Acknowledgements}

J.J., H.Z., and F.M. contributed equally to this work. This work was supported by the National Key Research and Development Program of China (2016YFA0100900), National Natural Science Foundation of China (No. 81671748), the National Key Basic Research Program of China (2014CB744504), the Project of Chinese Thousand Youth Talents. Prof. H. Zhang acknowledges Jane and Aatos Erkko Foundation (Grant no. 4704010), Academic of Finland (Grant no. 297580) and Sigrid Juselius Foundation (Grant no. 28001830K1) for financial support. Prof. H. A. Santos acknowledges financial support from the University of Helsinki Research Funds, the Sigrid Juselius Foundation (grant no. 4704580), the HiLIFE Research Funds, and the European Research Council under the European Union's Seventh Framework Programme (FP/2007-2013; grant no. 310892).

Received: ((will be filled in by the editorial staff))

Revised: ((will be filled in by the editorial staff)) Published online: ((will be filled in by the editorial staff)) 


\section{WILEY-VCH}

References

[1] C. E. DeSantis, J. Ma, A. G. Sauer, L. A. Newman, A. Jemal, CA-A Cancer J. Clin. 2017, $67,439$.

[2] N. Harbeck, M. Gnant, Lancet 2017, 389, 1134.

[3] L. Carey, E. Winer, G. Viale, D. Cameron, L. Gianni, Nat. Rev. Clin. Oncol. 2010, 7, 683.

[4] W. D. Foulkes, I. E. Smith, J. S. Reis, N. Engl. J. Med. 2010, 363, 1938.

[5] G. Bianchini, J. M. Balko, I. A. Mayer, M. E. Sanders, L. Gianni, Nat. Rev. Clin. Oncol. 2016, 13, 674 .

[6] P. Shah, A. Roth, R. Goya, A. Oloumi, G. Ha, Y. J., C. Caldas, M. A. Marra, S. Aparicio, Nature 2012, 486, 395.

[7] B. D. Lehmann, J. A. Bauer, X. Chen, M. E. Sanders, A. B. Chakravarthy, Y. Shyr, J. A. Pietenpol, J. Clin. Invest. 2011, 121, 2750.

[8] H. Kennecke, R. Yerushalmi, R. Woods, M. C. U. Cheang, D. Voduc, C. H. Speers, T. O. Nielsen, K. Gelmon, J. Clin. Oncol. 2010, 28, 3271.

[9] J. A. Joyce, J. W. Pollard, Nat. Rev. Cancer 2009, 9, 239.

[10] R. D. Loberg, D. A. Bradley, S. A. Tomlins, M. Chinnaiyan, K. J. Pieta, CA-A Cancer J. Clin. 2007, 57, 225.

[11] O. Metzger, A. Tutt, E. de Azambuja, K. S. Saini, G. Viale, S. Loi, I. Bradbury, J. M. Bliss, H. A. Azim, P. Ellis, A. Di Leo, J. Baselga, C. Sotiriou, M. Piccart-Gebhart, J. Clin. Oncol. 2012, 30, 1879.

[12] C. A. Hudis, L. Gianni, Oncologist 2011, 16, 1.

[13] P. K. Singal, N. Iliskovic, N. Engl. J. Med. 1998, 339, 900.

[14] G. Curigliano, D. Cardinale, S. Dent, C. Criscitiello, O. Aseyev, D. Lenihan, C. M. Cipolla, CA-A Cancer J. Clin. 2016, 66, 310.

[15] O. Tacar, P. Sriamornsak, C. R. Dass, J. Pharm. Pharmacol.y 2013, 65, 157.

[16] S. Zhang, X. Liu, T. Bawa-Khalfe, L.-S. Lu, Y. L. Lyu, L. F. Liu, E. T. H. Yeh, Nat. Med. 2012, 18, 1639.

[17] H. Sun, Q. Meng, S. Tang, J. Su, Q. Yin, L. Chen, W. Gu, H. Yu, Z. Zhang, S. Wang, Y. Li, Mol. Pharm. 2015, 12, 3323.

[18] E. Mastria, M. Chen, J. McDaniel, X. Li, J. Hyun, M. Dewhirst, A. Chikoti, J. Control. Release 2015, 208, 52.

[19] M. E. Davis, Z. Chen, D. M. Shin, Nat. Rev. Drug Discov. 2008, 7, 771.

[20] H. Wang, Y. Li, H. Bai, J. Shen, X. Chen, Y. Ping, G. Tang, Adv. Funct. Mater. 2017, 27 , 1700339. 


\section{WILEY-VCH}

[21] H. Wang, Y. Li, M. Zhang, Y. Shen, G. Tang, Y. Ping, Adv. Healthcare. Mater. 2017, 1601293.

[22] R. K. Jain, T. Stylianopoulos, Nat. Rev. Clin. Oncol. 2010, 7, 653.

[23] E. Blanco, H. Shen, M. Ferrari, Nat. Biotechnol 2015, 33, 941.

[24] H. Li, J. Du, X. Du, C. Xu, C. Sun, H. Wang, Z. Cao, X. Yang, Y. Zhu, S. Nie, J. Wang, Proc. Natl. Acad. Sci. USA 2016, 113, 4164.

[25] H. S. Choi, W. Liu, P. Misra, E. Tanaka, J. P. Zimmer, B. I. Ipe, M. G. Bawendi, J. V. Frangioni, Nat. Biotechnol. 2007, 25, 1165.

[26] S. Barua, S. Mitragotri, Nano Today 2014, 9, 223.

[27] M. Zhou, J. J. Li, S. Liang, A. K. Sood, D. Liang, C. Li, ACS Nano 2015, 9, 7085.

[28] M. R. Dreher, W. G. Liu, C. R. Michelich, M. W. Dewhirst, F. Yuan, A. Chilkoti, J. Natl. Cancer Inst. 2006, 98, 335.

[29] D. Huo, S. Liu, C. Zhang, J. He, Z. Zhou, H. Zhang, Y. Hu, ACS Nano 2017, 11, 10159.

[30] H. Xia, F. Li, X. Hu, W. Park, S. Wang, Y. Jang, Y. Du, S. Baik, S. Cho, T. Kang, D. H. Kim, D. Ling, K. M. Hui, T. Hyeon, ACS Cent. Sci. 2016, 2, 802.

[31] X.Guo, X. Wei, Y. Jing, S. Zhou, Adv. Mater. 2015, 27, 6450.

[32] L. Li, W. Sun, J. Zhong, Q. Yang, X. Zhu, Z. Zhou, Z. Zhang, Y. Huang, Adv. Funct. Mater. 2015, 25, 4101.

[33] R. M. Barber, N. Fullman, R. J. D. Sorensen, T. J. Beyene, Lancet 2017, 390, 231.

[34] Y. Cheng, J. D. Meyers, A.-M. Broome, M. E. Kenney, J. P. Basilion, C. Burda, J. Am. Chem. Soc. 2011, 133, 2583.

[35] C. C. Hung, W.-C. Huang, Y. W. Lin, T. W. Yu, H. H. Chen, S. C. Lin, W. H. Chiang, H. C. Chiu, Theranostics 2016, 6, 302.

[36] S. Ruan, X. Cao, X. Cun, G. Hu, Y. Zhou, Y. Zhang, L. Lu, Q. He, H. Gao, Biomaterials 2015, 60,100 .

[37] H. Cabral, Y. Mats umoto, K. Mizuno, Q. Chen, M. Murakami, M. Kimura, Y. Terada, M. R. Kano, K. Miyazono, M. Uesaka, N. Nishiyama, K. Kataoka, Nat. Nanotechnol. 2011, 6, 815 .

[38] L. H. V. Wang, S. Hu, Science 2012, 335, 1458.

[39] C. Kim, C. Favazza, L. H. V. Wang, Chem. Rev. 2010, 110, 2756-2782.

[40] F. Chen, H. Hong, Y. Zhang, H. F. Valdovinos, S. Shi, G. S. Kwon, C. P. Theuer, T. E. Barnhart, W. Cai, ACS Nano 2013, 7, 9027.

[41] H. Hong, K. Yang, Y. Zhang, J. W. Engle, L. Feng, Y. Yang, T. R. Nayak, S. Goel, J. Bean, C. P. Theuer, T. E. Barnhart, Z. Liu, W. Cai, ACS Nano 2012, 6, 2361. 


\section{WILEY-VCH}

[42] G. Sun, J. Xu, A. Hagooly, R. Rossin, Z. Li, D. A. Moore, C. J. Hawker, M. J. Welch, K. L. Wooley, Adv. Mater. 2007, 19, 3157.

[43] T. Tanei, F. Leonard, X. Liu, J. F. Alexander, Y. Saito, M. Ferrari, B. Godin, K. Yokoi, Cancer Res. 2016, 76, 429.

[44] Q. Li, Z. Ma, Y. Liu, X. Kan, C. Wang, B. Su, Y. Li, Y. Zhang, P. Wang, Y. Luo, D. Na, L. Wang, G. Zhang, X. Zhu, L. Wang, FEBS J. 2016, 283, 2836.

[45] H. J. Paholak, N. O. Stevers, H. Chen, J. P. Burnett, M. He, H. Korkaya, S. P. McDermott, Y. Deol, S. G. Clouthier, T. Luther, Q. Li, M. S. Wicha, D. Sun, Biomaterials 2016, 104, 145.

[46] D. Samanta, D. M. Gilkes, P. Chaturvedi, L. S. Xiang, G. L. Semenza, Proc. Natl. Acad. Sci. USA 2014, 111, E5429.

[47] R. Z. Atkinson, M. Diagaradjane, P. Peddibhotla, S. Contreras, A. Hilsenbeck, S. Woodward, W. Krishnan, S. Chang, J. Rosen, J, Sci. Transl. Med. 2010, 2, 55 ra79. 


\section{WILEY-VCH}

A novel tumor penetrating multifunctional nanosystem for triple negative breast cancer combination therapy under photoacoustic images guidance was developed. The nanosystem possesses neglect cardiotoxicity, can improve the drug penetration depth, eradicate breast cancer stem cells, and inhibit lung and liver metastasis.

Keyword: triple-negative breast cancer, nanoparticles, self-assembly, tumor penetration, metastasis

Jianfeng Ji, Fei Ma, Hongbo Zhang, Fengyong Liu, Jian He, Wanlin Li, Tingting Xie, Danni Zhong, Tingting Zhang, Mei Tian,* Hong Zhang,* Hélder A. Santos,* and Min Zhou*

\section{Non-Cardiotoxic Tumor-Penetrating Nanosystem for Triple Negative Breast Cancer to Eradicate Lung and Liver Metastasis}

\section{ToC figure}

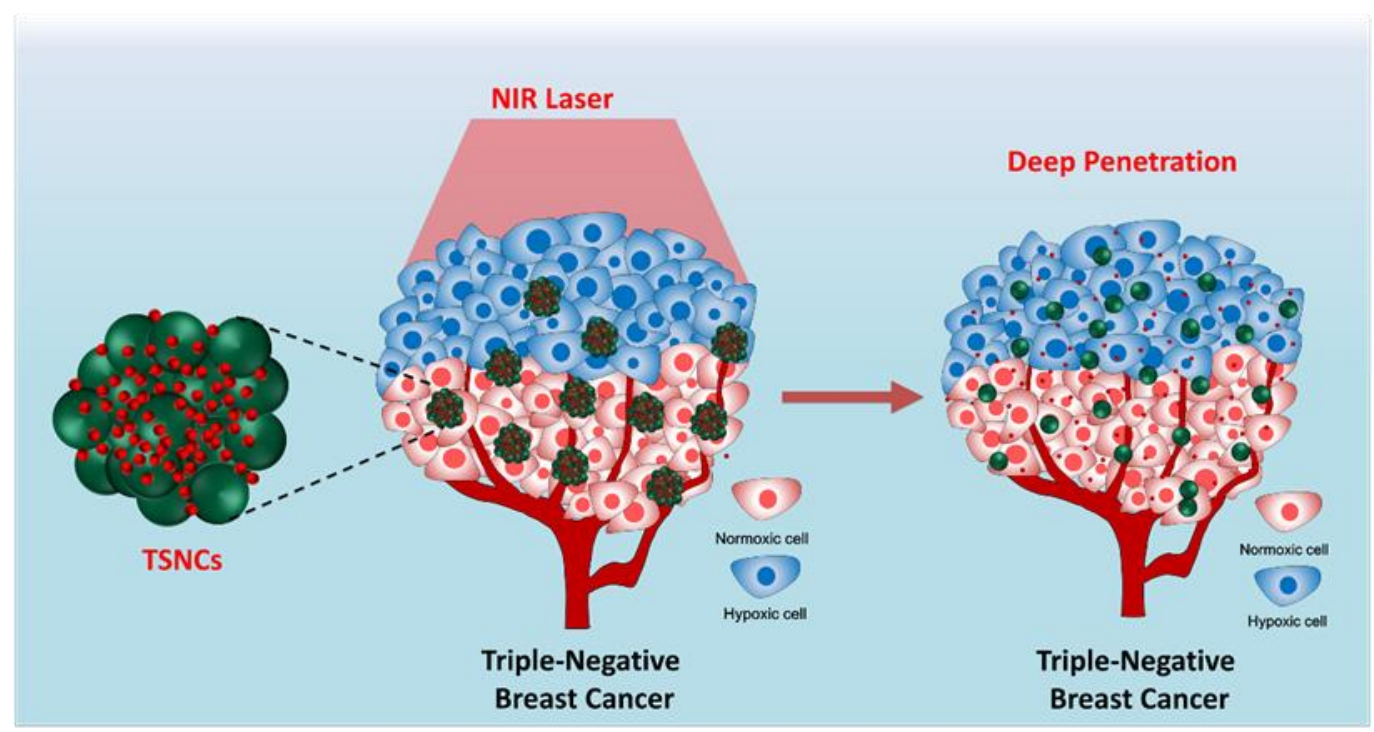




\section{Click here to access/download \\ Supporting Information
AFM TSNCs Supp-Rev 2.doc \\ Supporting Information
AFM TSNCs Supp-Rev 2.doc}


Fig. 1

\title{
Click here to access/download \\ Production Data Figure 1.tif

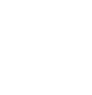

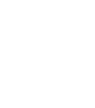

if
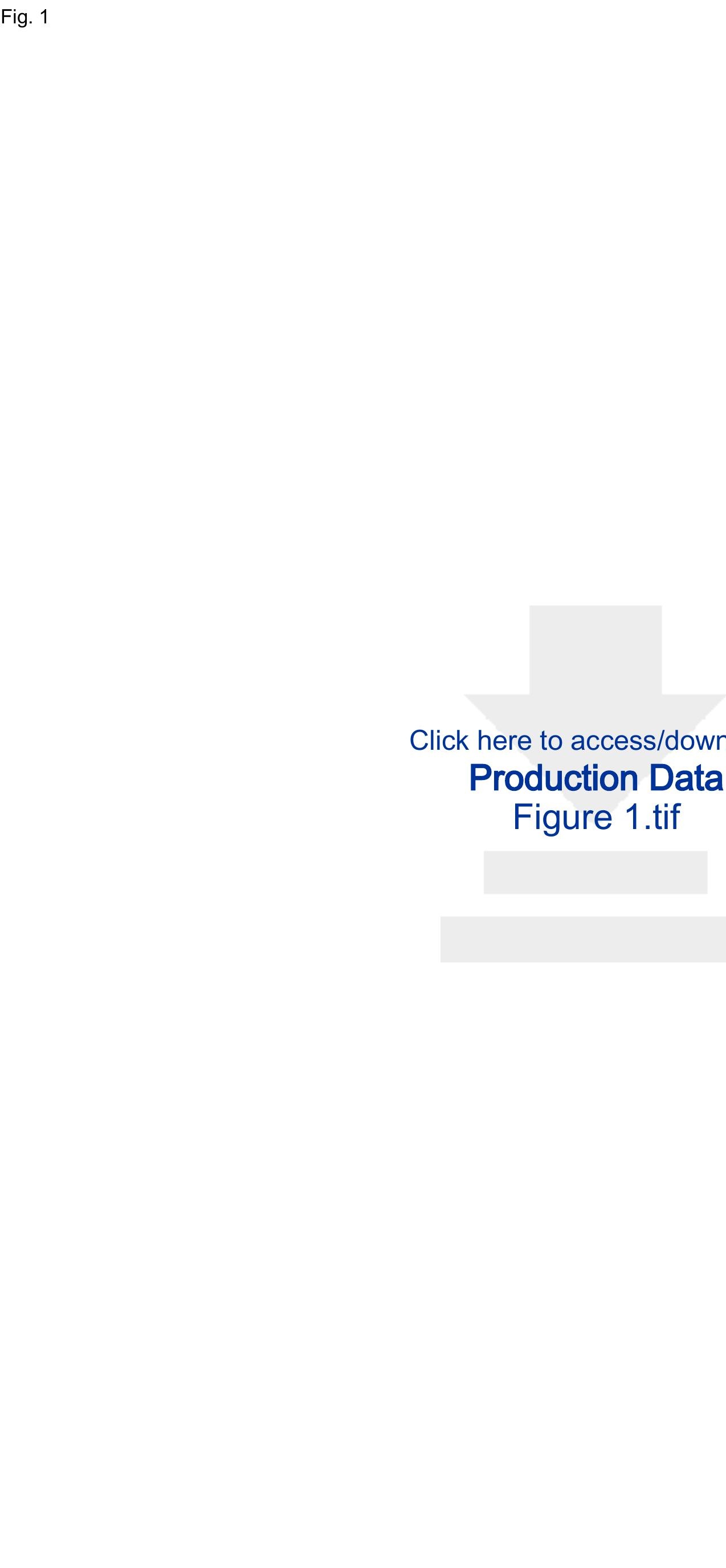
Fig. 2

\section{Click here to access/download \\ Production Data Figure 2.tif$$
\text { . }
$$

(a)

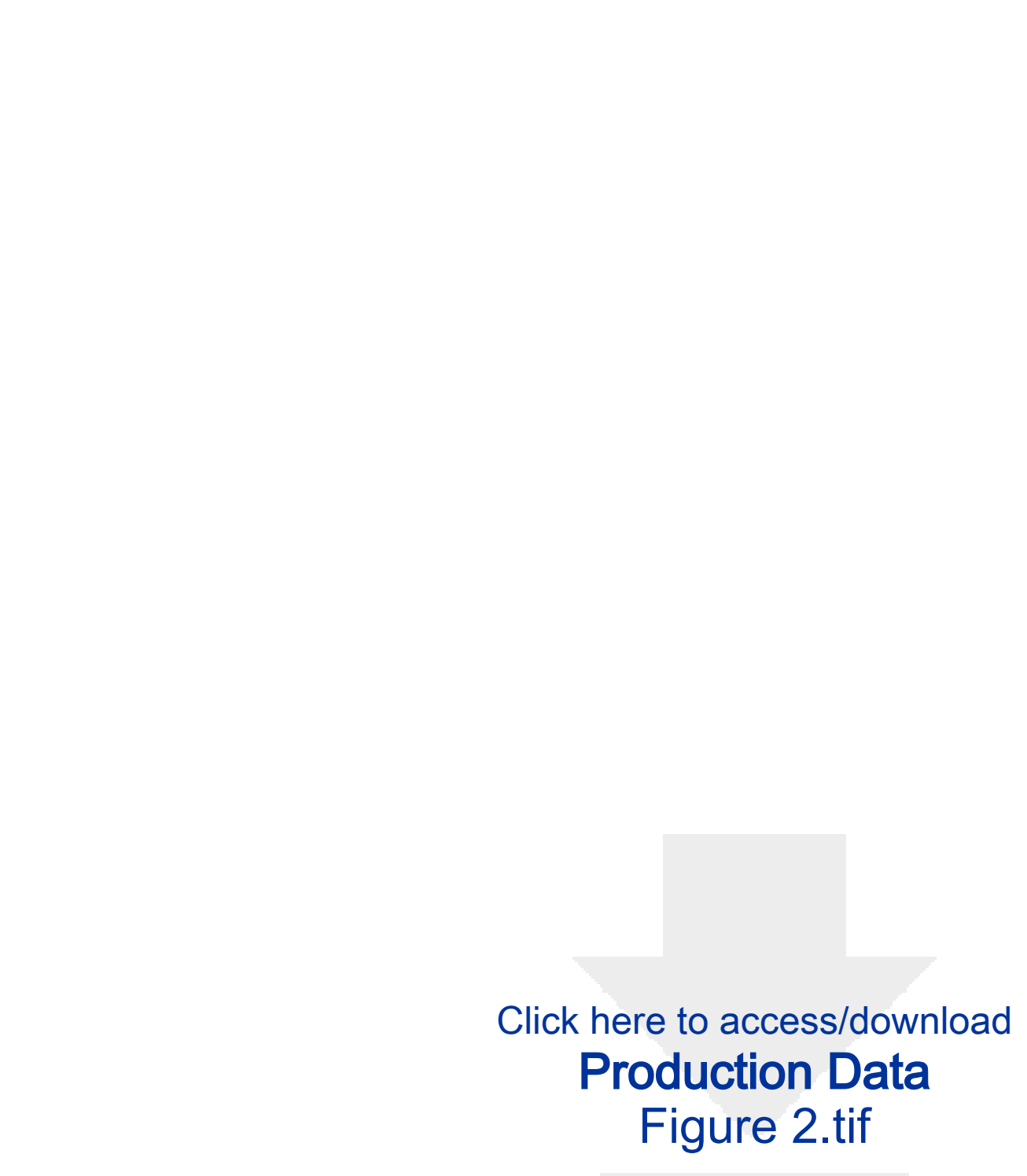

.

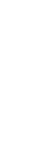

(1) 
Fig. 3

\section{Click here to access/download
Production Data \\ Click here to access/download
Production Data

\author{
Figure 3.tif

\begin{abstract}
Figure 3.4 .
\end{abstract}

(
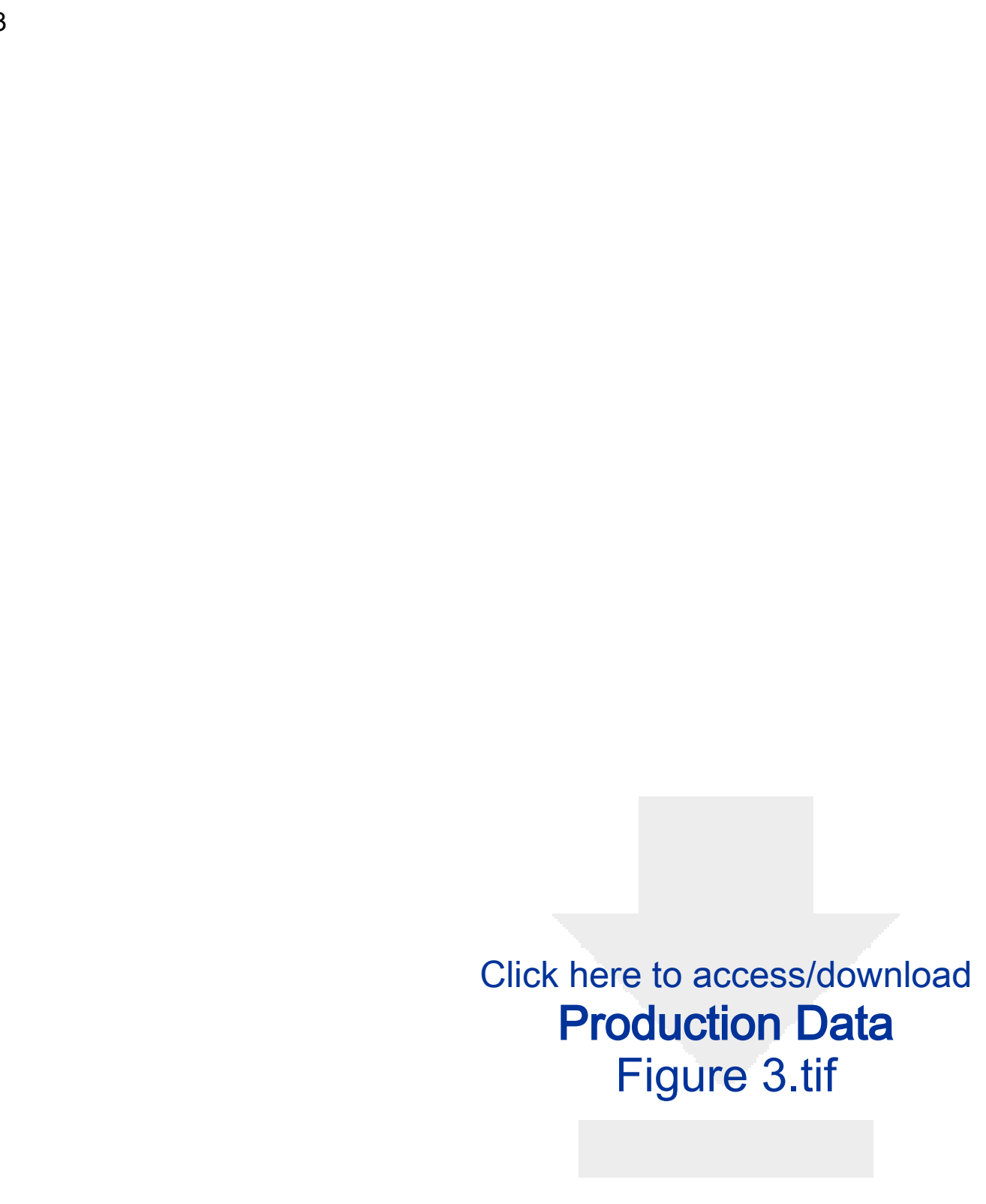

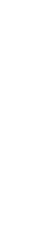


Fig. 4
\[ \begin{array}{c}\text { Click here to access/download } \\ \text { Production Data }\end{array} \]

Fig. 4
\[ \begin{array}{c}\text { Production Data } \\ \text { Click here to access/downlo }\end{array} \]

Figure 4.tif

Fig. 4 Production Data
Click here to access/downlo

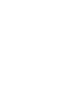

Fig. 4
\[ \begin{array}{c}\text { Click here to access/download } \\ \text { Production Data } \\ \text { Figure 4.tif }\end{array} \]

Figure 4 .tif

Figure 4.tif

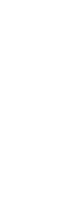


Fig. 5

\section{Click here to access/download \\ Production Data Figure 5.tif

a

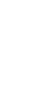
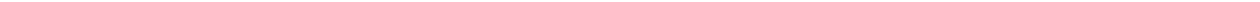
Fig. 6

6

.

- 
Fig. 7

\section{Click here to access/download
Production Data \\ Click here to access/download
Production Data
Figure 7.tif Production Data
Figure 7.tif Production Data
Figure 7.tif}

.

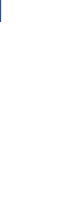

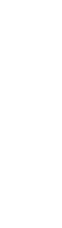

(1) 
Production Data
Figure 10.tif
Click here to access/download
Prota Production Data
Figure 10.tif
Click here to access/download
Protign

Production Data
Figure 10.tif
Click here to access/download
Proda

Fig. 10

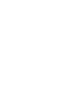

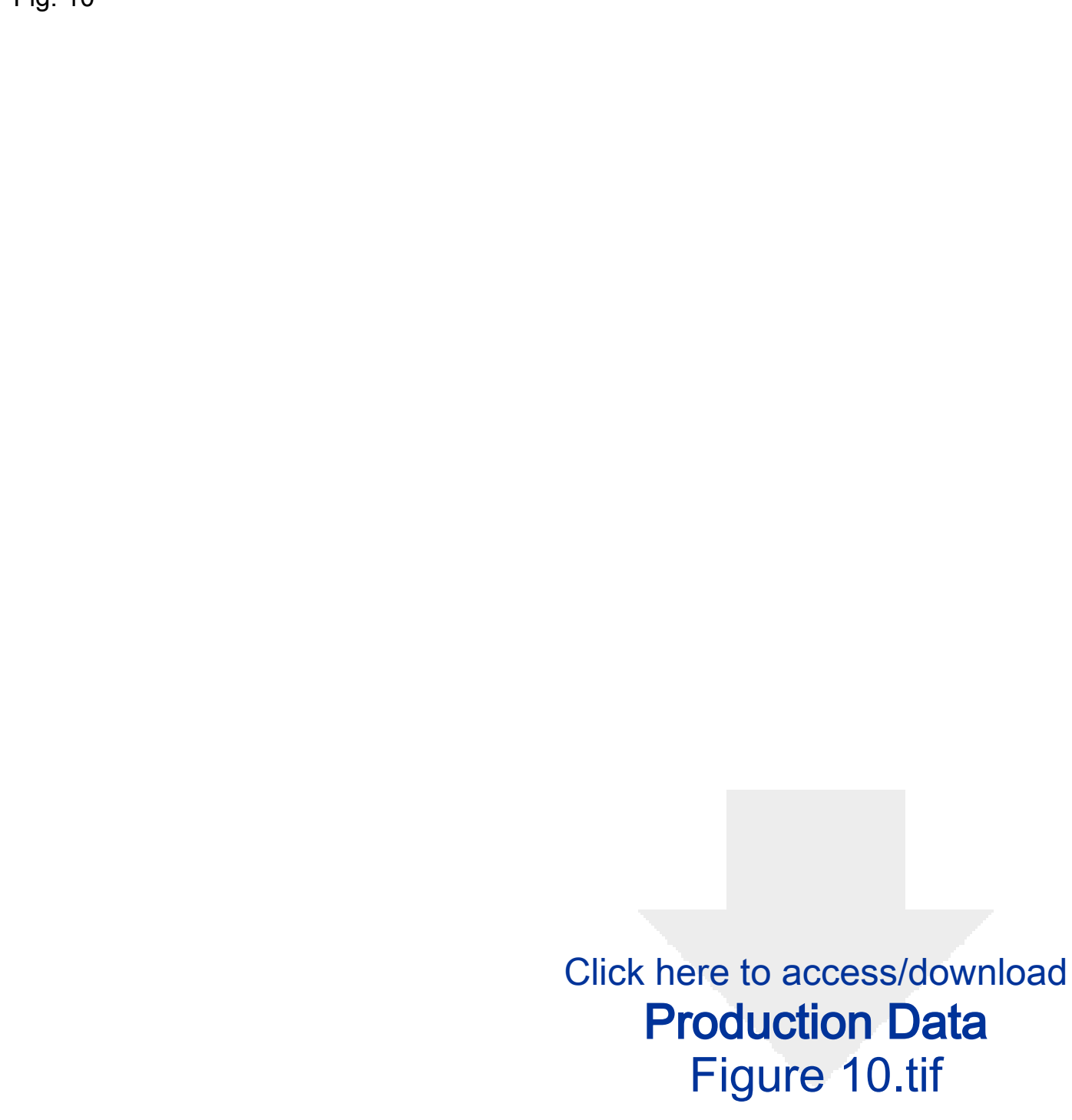
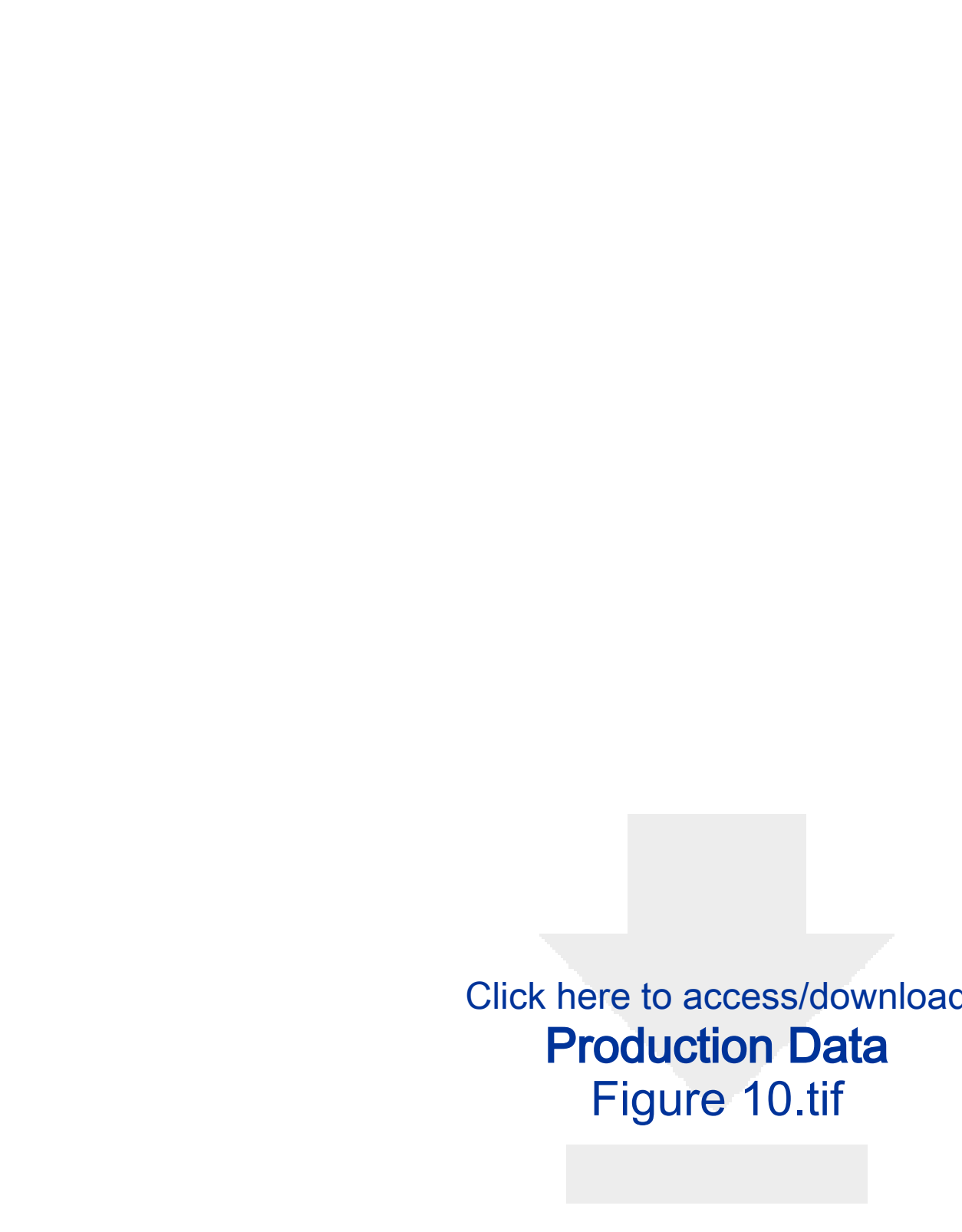

.

(1)

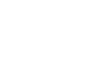

.

.

.

.

.

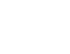

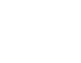

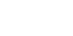

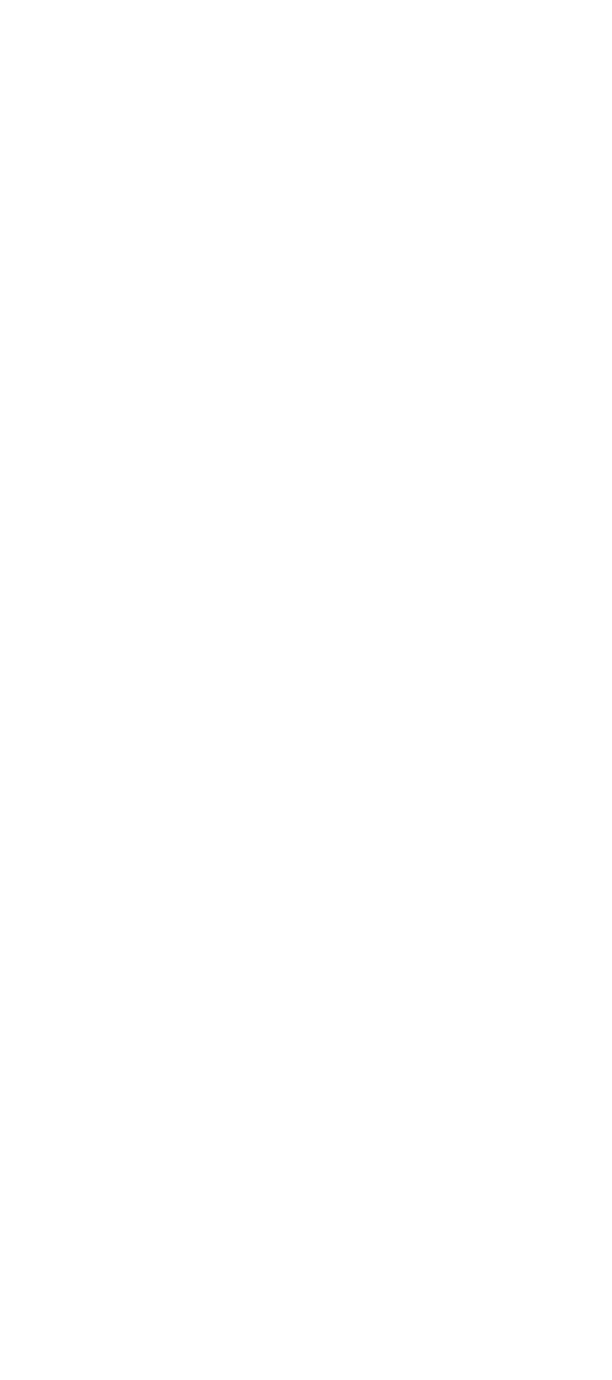

.

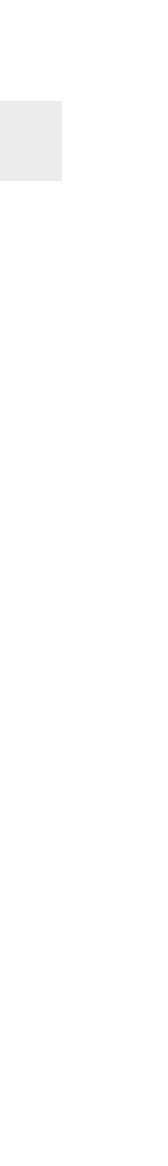


Fig. 8

\section{Click here to access/download
Production Data \\ Click here to access/download
Production Data Figure 8.tif

(

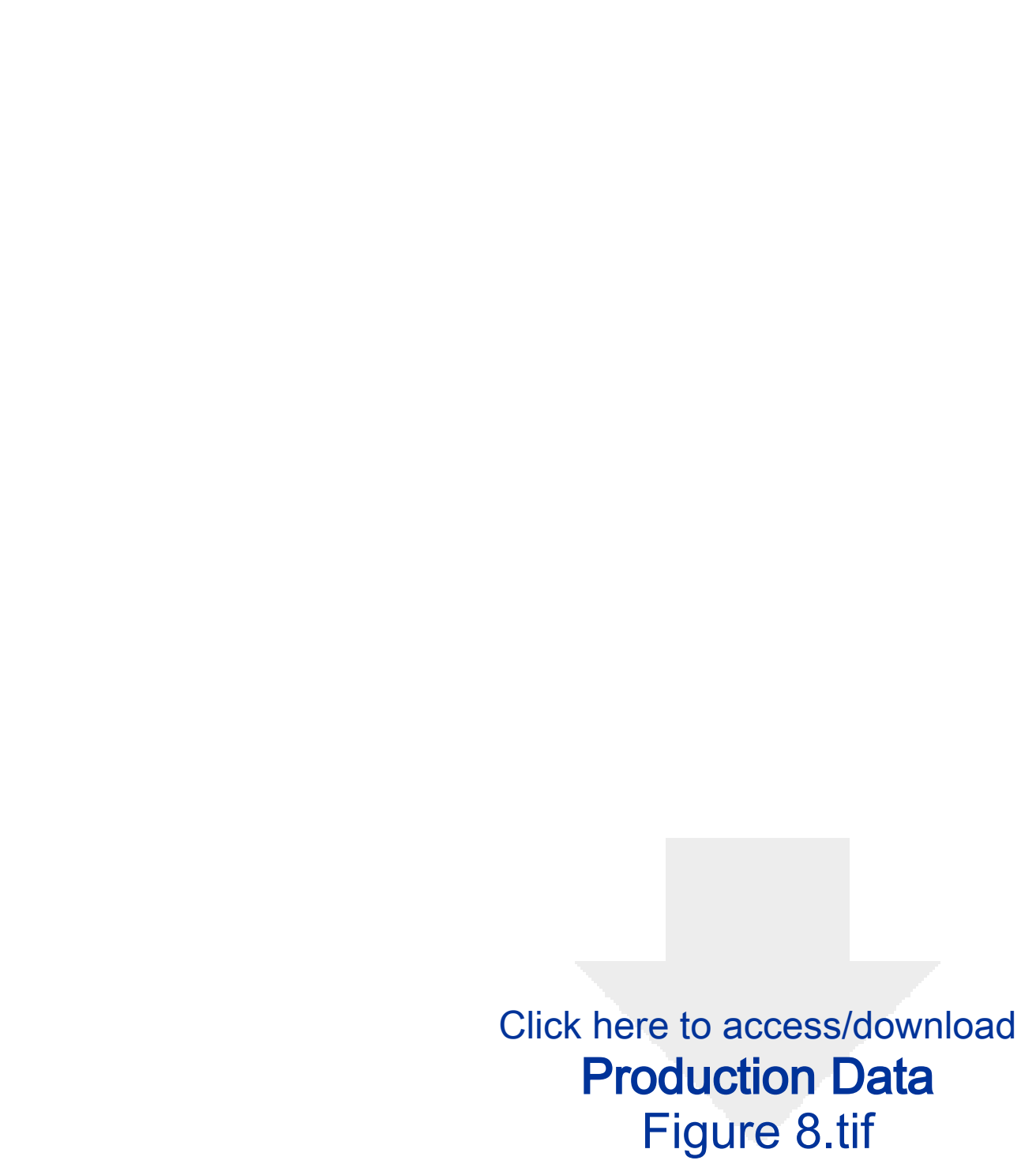


Graphical Abstract

Click here to access/download Production Data Graphic Abstract.tif 